\title{
Critical currents in superconductors with quasiperiodic pinning arrays: One-dimensional chains and two-dimensional Penrose lattices
}

\author{
Vyacheslav Misko ${ }^{1,2}$, Sergey Savel'ev ${ }^{1}$, and Franco Nori ${ }^{1,2}$ \\ ${ }^{1}$ Frontier Research System, The Institute of Physical and Chemical \\ Research (RIKEN), Wako-shi, Saitama, 351-0198, Japan and \\ ${ }^{2}$ Center for Theoretical Physics, Center for the Study of Complex Systems, \\ Department of Physics, University of Michigan, Ann Arbor, MI 48109-1040, USA
}

(Dated: October 26, 2018)

\begin{abstract}
We study the critical depinning current $J_{c}$, as a function of the applied magnetic flux $\Phi$, for quasiperiodic (QP) pinning arrays, including one-dimensional (1D) chains and two-dimensional (2D) arrays of pinning centers placed on the nodes of a five-fold Penrose lattice. In 1D QP chains of pinning sites, the peaks in $J_{c}(\Phi)$ are shown to be determined by a sequence of harmonics of long and short periods of the chain. This sequence includes as a subset the sequence of successive Fibonacci numbers. We also analyze the evolution of $J_{c}(\Phi)$ while a continuous transition occurs from a periodic lattice of pinning centers to a $\mathrm{QP}$ one; the continuous transition is achieved by varying the ratio $\gamma=a_{S} / a_{L}$ of lengths of the short $a_{S}$ and the long $a_{L}$ segments, starting from $\gamma=1$ for a periodic sequence. We find that the peaks related to the Fibonacci sequence are most pronounced when $\gamma$ is equal to the "golden mean". The critical current $J_{c}(\Phi)$ in QP lattice has a remarkable self-similarity. This effect is demonstrated both in real space and in reciprocal $k$-space. In $2 \mathrm{D}$ QP pinning arrays (e.g., Penrose lattices), the pinning of vortices is related to matching conditions between the vortex lattice and the QP lattice of pinning centers. Although more subtle to analyze than in 1D pinning chains, the structure in $J_{c}(\Phi)$ is determined by the presence of two different kinds of elements forming the 2D QP lattice. Indeed, we predict analytically and numerically the main features of $J_{c}(\Phi)$ for Penrose lattices. Comparing the $J_{c}$ 's for QP (Penrose), periodic (triangular) and random arrays of pinning sites, we have found that the QP lattice provides an unusually broad critical current $J_{c}(\Phi)$, that could be useful for practical applications demanding high $J_{c}$ 's over a wide range of fields.
\end{abstract}

PACS numbers: $74.25 . \mathrm{Qt}$

\section{INTRODUCTION}

Recent progress in the fabrication of nanostructures has provided a wide variety of well-controlled vortexconfinement topologies, including different types of regular pinning arrays. A main fundamental question in this field is how to drastically increase vortex pinning, and thus the critical current $J_{c}$, using artificially-produced periodic arrays of pinning sites (APS). These periodic APS have been extensively used for studying vortex pinning and vortex dynamics. In particular, enhanced $J_{c}$ and commensurability effects have been demonstrated in superconducting thin films with square and triangular arrays of sub- $\mu \mathrm{m}$ holes (i.e., antidots) $[1,2$, , 3, 4, 5, [6]. Moreover, blind antidots (i.e., holes which partially perforate the film to a certain depth) [], or pinning arrays with field-dependent pinning strength [8], provide more flexibility for controlling properties such as pinning strength, anisotropy, etc. The increase and, more generally, control of the critical current $J_{c}$ in superconductors by its patterning (perforation) can be of practical importance for applications in micro- and nanoelectronic devices.

A peak in the critical current $J_{c}(\Phi)$, for a given value of the magnetic flux, say $\Phi_{1}$, can be engineered using a superconducting sample with a periodic APS with a matching field $H_{1}=\Phi_{1} / A$ (where $A$ is the area of the pinning cell), corresponding to one trapped vortex per pinning site. However, this peak in $J_{c}(\Phi)$, while useful to obtain, decreases very quickly for fluxes away from $\Phi_{1}$. Thus, the desired peak in $J_{c}(\Phi)$ is too narrow and not very robust against changes in $\Phi$. It would be greatly desirable to have samples with APS with many periods. This multiple-period APS sample would provide either very many peaks or an extremely broad peak in $J_{c}(\Phi)$, as opposed to just one (narrow) main peak (and its harmonics). We achieve this goal (a very broad $J_{c}(\Phi)$ ) here by studying samples with many built-in periods.

The development of new fabrication technologies for pinning arrays with controllable parameters allows to fabricate not only periodic (square or triangular) but also more complicated quasiperiodic (QP) arrays of pinning sites, including Penrose lattices [9, 10, 11].

The investigation of physical properties of QP systems has attracted considerable interest including issues such as band structure and localization of electronic states in two-dimensional (2D) Penrose lattice [12, 13], electronic and acoustic properties of one-dimensional (1D) QP lattices 14, 15], superconducting-to-normal phase boundaries of 2D QP micronetworks [16, 17, 18, 19, 20], QP semiconductor heterostructures and optical superlattices 21], soliton pinning by long-range order 22] and pulse propagation [23] in QP systems. Moreover, increasing and, more generally, controlling the critical current in superconductors by its patterning (perforation) can be of practical importance for applications in micro- and 
nanoelectronic devices.

The original tiling has been studied in Ref. [9]. The inflation (or production) rules of "finite Penrose patterns" generated by repeated application of deflation and rescaling have been found, which show a definite hierarchical structure of the Penrose patterns [1].

The electronic and acoustic properties of a onedimensional quasicrystal have been studied in Refs. 13, 14]. It has been shown, in particular, that there exist two types of the wave functions, self-similar (fractal) and nonself-similar (chaotic) which show "critical" or "exotic" behavior [13]. By both numerical (non-perturbative) and analytical (perturbative) approaches, it has been demonstrated [14, 15] that the phonon and electronic spectra of 1D quasicrystals exhibit a self-similar hierarchy of gaps and localized states in the gaps. The existence of gaps, and gap states, in QP GaAs-AlGaAs superlattices has been predicted and found experimentally.

Along with studying the structural, electronic and acoustic properties of QP structures, considerable progress has been reached in understanding the superconducting properties of $2 \mathrm{D}$ quasicrystalline arrays 16, 17, 18, 19, 20]. The effect of frustration, induced by a magnetic field, on the superconducting diamagnetic properties has been revealed and the superconductingto-normal phase boundaries, $T_{c}(H)$, have been calculated for several geometries with quasicrystalline order, in a good agreement with experimentally measured phase boundaries 17, 19]. A comprehensive analysis of superconducting wire networks including quasicrystalline geometries and Josephson-junction arrays in a magnetic field has been presented in Refs 19, 20]. An analytical approach [19, 20] was introduced to analyze the structures which are present in phase diagrams for a number of geometries. It has been shown that the gross structure is determined by the statistical distributions of the cell areas, and that the fine structures are determined by correlations among neighboring cells in the lattices. The effect of thermal fluctuations on the structure of the phase diagram has been studied [19] by a cluster mean-field calculation and using real-space renormalization group.

In this paper, we study another phenomenon related to superconducting properties of quasiperiodic systems, namely, vortex pinning by $1 \mathrm{D}$ QP chains and by $2 \mathrm{D}$ arrays of pinning sites located at the nodes of QP lattices (e.g., a five-fold Penrose lattice). It should be noted that in superconducting networks the areas of the network plaquettes play a dominant role [16, 17, 18, 19]. However, for vortex pinning by QP pinning arrays, the specific geometry of the elements which form the QP lattice and their arrangement (and not just the areas) are important, making the problem complicated.

In Sec. II we introduce the model used for describing vortex dynamics in QP pinning arrays and for determining the critical depinning current, $J_{c}$, which is analyzed for different quasicrystalline geometries.

The pinning of vortices by a 1D QP chain of pinning sites (i.e., Fibonacci sequence) is discussed in Sec. III. We consider a continuous transition from a periodic to the QP chain of pinning sites and we monitor the corresponding changes in the critical current $J_{c}$ as a function of the applied magnetic flux $\Phi$. A remarkable self-similarity of $J_{c}(\Phi)$ is demonstrated in both real space and in reciprocal $k$-space.

Section IV studies the pinning of flux lattices by 2D QP pinning arrays including the five-fold Penrose lattice. We analyze changes of the function $J_{c}(\Phi)$ during a continuous transition from a periodic triangular lattice of pinning sites to the Penrose lattice. Based on detailed considerations of the structure and specific local rules of construction of the Penrose lattice, we predict the main features of the function $J_{c}(\Phi)$. Numerical simulations with different finite-size Penrose lattices confirm the predicted main features for large-size lattices, that is important for possible experimental observations of the revealed quasiperiodic features. We also obtain analytical results supporting our conclusions. Moreover, we also discuss the changes in the critical current by adding either a "quasiperiodic" modulation or random displacements to initially periodic pinning arrays.

\section{MODEL}

We model a three-dimensional (3D) slab infinitely long in the $z$-direction, by a two-dimensional (2D) (in the $x y$ plane) simulation cell with periodic boundary conditions, assuming the vortex lines are parallel to the cell edges. To study the dynamics of moving vortices driven by a Lorentz force, interacting with each other and with pinning centers, we perform simulated annealing simulations by numerically integrating the overdamped equations of motion (see, e.g., Ref. 24, 25, 26, 27]):

$$
\eta \mathbf{v}_{i}=\mathbf{f}_{i}=\mathbf{f}_{i}^{v v}+\mathbf{f}_{i}^{v p}+\mathbf{f}_{i}^{T}+\mathbf{f}_{i}^{d} .
$$

Here, $\mathbf{f}_{i}$ is the total force per unit length acting on vortex $i, \mathbf{f}_{i}^{v v}$ and $\mathbf{f}_{i}^{v p}$ are the forces due to vortex-vortex and vortex-pin interactions, respectively, $\mathbf{f}_{i}^{T}$ is the thermal stochastic force, and $\mathbf{f}_{i}^{d}$ is the driving force acting on the $i$-th vortex; $\eta$ is the viscosity, which is set to unity. The force due to the interaction of the $i$-th vortex with other vortices is

$$
\mathbf{f}_{i}^{v v}=\sum_{j}^{N_{v}} f_{0} K_{1}\left(\frac{\left|\mathbf{r}_{i}-\mathbf{r}_{j}\right|}{\lambda}\right) \hat{\mathbf{r}}_{i j},
$$

where $N_{v}$ is the number of vortices, $K_{1}$ is a modified Bessel function, $\lambda$ is the magnetic field penetration depth, $\hat{\mathbf{r}}_{i j}=\left(\mathbf{r}_{i}-\mathbf{r}_{j}\right) /\left|\mathbf{r}_{i}-\mathbf{r}_{j}\right|$, and

$$
f_{0}=\frac{\Phi_{0}^{2}}{8 \pi^{2} \lambda^{3}} .
$$

Here $\Phi_{0}=h c / 2 e$ is the magnetic flux quantum. It is convenient, following the notation used in Ref. 24, 25, [26, 27], to express all the lengths in units of $\lambda$ and all 
the fields in units of $\Phi_{0} / \lambda^{2}$. The Bessel function $K_{1}(r)$ decays exponentially for $r$ greater than $\lambda$, therefore it is safe to cut off the (negligible) force for distances greater than $5 \lambda$. The logarithmic divergence of the vortex-vortex interaction forces for $r \rightarrow 0$ is eliminated by using a cutoff for distances less than $0.1 \lambda$.

Vortex pinning is modeled by short-range parabolic potential wells located at positions $\mathbf{r}_{k}^{(p)}$. The pinning force is

$$
\mathbf{f}_{i}^{v p}=\sum_{k}^{N_{p}}\left(\frac{f_{p}}{r_{p}}\right)\left|\mathbf{r}_{i}-\mathbf{r}_{k}^{(p)}\right| \Theta\left(\frac{r_{p}-\left|\mathbf{r}_{i}-\mathbf{r}_{k}^{(p)}\right|}{\lambda}\right) \hat{\mathbf{r}}_{i k}^{(p)},
$$

where $N_{p}$ is the number of pinning sites, $f_{p}$ is the maximum pinning force of each potential well, $r_{p}$ is the range of the pinning potential, $\Theta$ is the Heaviside step function, and $\hat{\mathbf{r}}_{i k}^{(p)}=\left(\mathbf{r}_{i}-\mathbf{r}_{k}^{(p)}\right) /\left|\mathbf{r}_{i}-\mathbf{r}_{k}^{(p)}\right|$.

The temperature contribution to Eq. (11) is represented by a stochastic term obeying the following conditions:

$$
\left\langle f_{i}^{T}(t)\right\rangle=0
$$

and

$$
\left\langle f_{i}^{T}(t) f_{j}^{T}\left(t^{\prime}\right)\right\rangle=2 \eta k_{B} T \delta_{i j} \delta\left(t-t^{\prime}\right) .
$$

The ground state of a system of moving vortices is obtained as follows. First, we set a high value for the temperature, to let vortices move randomly. Then, the temperature is gradually decreased down to $T=0$. When cooling down, vortices interacting with each other and with the pinning sites adjust themselves to minimize the energy, simulating the field-cooled experiments [29, 30].

In order to find the critical depinning current, $J_{c}$, we apply an external driving force gradually increasing from $f_{d}=0$ up to a certain value $f_{d}=f_{d}^{c}$, at which all the vortices become depinned and start to freely move. For values of the driving force just above $f_{d}^{c}$, the total current of moving vortices $J \sim\langle v\rangle$ becomes nonzero. Here, $\langle v\rangle$ is the normalized (per vortex) average velocity of all the vortices moving in the direction of the applied driving force. In numerical simulations, this means that, in practice, one should define some threshold value $J_{\text {min }}$ larger than the noise level. Values larger than $J_{\min }$ are then considered as nonzero currents. However, instead using this criterion-sensitive scheme, we can use an alternative approach based on potential energy considerations. In case of deep short-range potential wells, the energy required to depin vortices trapped by pinning sites is proportional to the number of pinned vortices, $N_{v}^{(p)}$. Therefore, in this approximation we can define the "critical current" as follows:

$$
J_{c}(\Phi)=J_{0} \frac{N_{v}^{(p)}(\Phi)}{N_{v}(\Phi)}
$$

where $J_{0}$ is a constant, and study the dimensionless value $J_{c}^{\prime}=J_{c} / J_{0}$ (further on, the primes will be omitted).
Throughout this work, we use narrow potential wells as pinning sites, characterized by $r_{p}=0.04 \lambda$ to $0.1 \lambda$. Our calculations show that, for the parameters used, the dependence of the critical current $J_{c}(\Phi)$ defined according to Eq. (6), is in good agreement to that based on the above general definition of $J_{c}$ (which involves an adjustable parameter $\left.J_{\min }\right)$. The advantages of using $J_{c}$ defined by Eq. (6) are the following: it (i) does not involve any arbitrary threshold $J_{\min }$ and (ii) is less CPUtime consuming, allowing the study of very large-size lattices. Moreover, the goal of this study is to reveal specific matching effects between a (periodic) vortex lattice and arrays of QP pinning sites, and to study how the quasiperiodicity manifests itself in experimentally measurable quantities $\left(J_{c}, N_{v}^{(p)} / N_{v}\right)$ related to the vortex pinning by QP (e.g., the Penrose lattice) pinning arrays.

\section{PINNING OF VORTICES BY A 1D QUASIPERIODIC CHAIN OF PINNING SITES}

In this section we study the pinning of vortices by one dimensional (1D) QP chains of pinning sites.

\section{A. 1D quasiperiodic chain}

As an example of a 1D QP chain, or 1D quasicrystal, a Fibonacci sequence is considered, which can be constructed following a simple procedure: let us consider two line segments, long and short, denoted, respectively, by $L$ and $S$. If we place them one by one, we obtain an infinite periodic sequence:

$$
\text { LSLSLSLSLSLSLSLS .. }
$$

A unit cell of this sequence consists of two elements, $L$ and $S$. In order to obtain a QP sequence, these elements are transformed according to Fibonacci rules as follows: $L$ is replaced by $L S, S$ is replaced by $L$ :

$$
L \rightarrow L S, \quad S \rightarrow L .
$$

As a result, we obtain a new sequence:

$$
\text { LSLLSLLSLLSLLSL ... }
$$

Iteratively applying the rule (8) to the sequence (9), we obtain, in the next iteration, a sequence with a fiveelement unit cell $(L S L L S)$, then with an eight-element unit cell $(L S L L S L S L)$, and so on, to infinity. For the sequence with an $n$-element cell, where $n \rightarrow \infty$, the ratio of numbers of long to short elements is the golden mean value,

$$
\tau=(1+\sqrt{5}) / 2 .
$$

The position of the $n$th point where a new element, either $L$ or $S$, begins is determined by [1]:

$$
x_{n}=n+\gamma[\gamma n],
$$




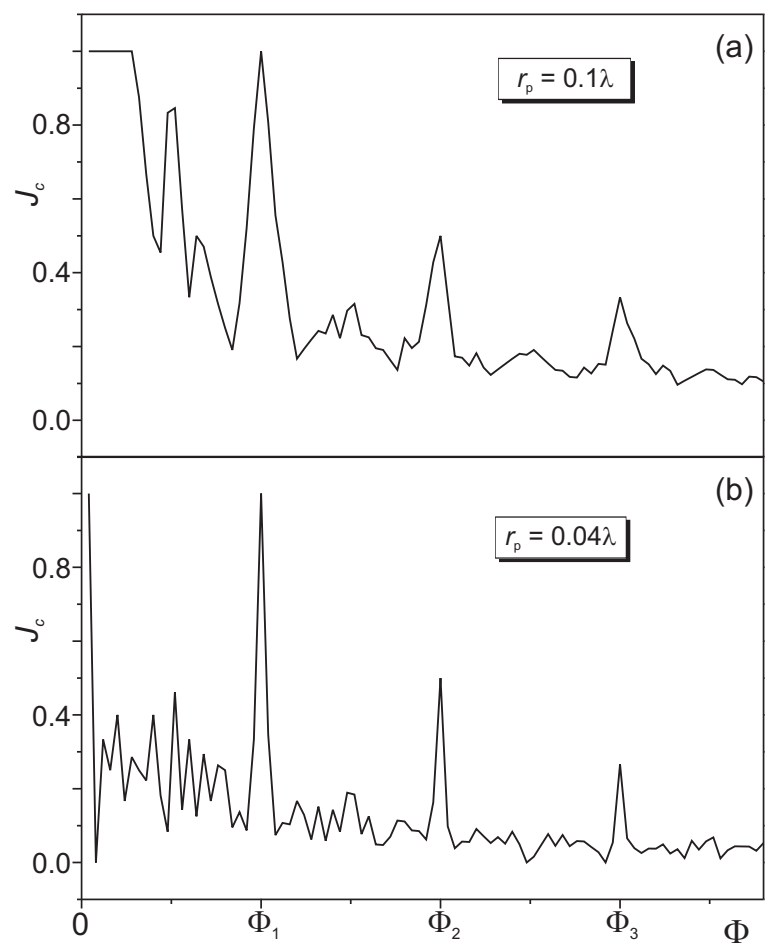

FIG. 1: (a, b) Dimensionless critical depinning current $J_{c}$, as a function of the applied magnetic flux $\Phi$, in a 1D periodic chain of pinning sites, for a cell containing 25 pinning sites, $N_{p}=25$ and $f_{p} / f_{0}=2.0$. The indicated fluxes $\Phi_{1}, \Phi_{2}$, and $\Phi_{3}$ correspond to the first, second, and third matching fields. The function $J_{c}(\Phi)$ is shown for two different values of the pinning site radius, $r_{p}=0.1 \lambda(\mathrm{a})$, and $r_{p}=0.04 \lambda$ (b). When $r_{p}$, decreases, the main commensurability peaks become sharper, as shown in (a) and (b).

where $[x]$ denotes the maximum integer less or equal to $x$. Equation (11) corresponds to the case when the Fibonacci sequence has a ratio $\gamma=a_{S} / a_{L}$ of the length of the short segment, $a_{S}$, to the length of the long segment, $a_{L}$. Ratios $\gamma$ other than $1 / \tau$ correspond to other chains which are all QP. Along with the golden mean value of $\gamma=1 / \tau$, we use in our simulations $\gamma$ 's varying in the interval between 0 and 1: $0<\gamma<1$, when analyzing a continuous transition from a periodic to a QP (Fibonacci sequence) pinning array.

To study the critical depinning current $J_{c}$ in $1 \mathrm{D}$ QP pinning chains, we place pinning sites on the points where $L$ or $S$ elements of the QP sequence link to each other. Therefore, the coordinates of the centers of the pinning sites are defined by Eq. (11) with $\gamma=a_{S} / a_{L}$.

\section{B. Pinning of vortices by a $1 \mathrm{D}$ periodic chain of pinning sites}

We start with a periodic chain of pinning sites, which can be considered, in the framework of the above scheme, as a limiting case of a "QP" chain with $\gamma=1$, i.e. $a_{S}=$ $a_{L}=1$. In Fig. 1a, the critical current $J_{c} \sim N_{v}^{(p)} / N_{v}$ is shown as a function of the applied magnetic flux $\Phi$ for $f_{p} / f_{0}=2$ and for a pinning site radius $r_{p}=0.1 \lambda$. Sharp peaks of the function $J_{c}(\Phi)$ correspond to matching fields. Since the dimensionless critical current we plot is that per vortex and is proportional to the number of pinned vortices and inversely proportional to the total number of vortices (Eq (6) ), therefore, the magnitude of the peaks versus $\Phi \sim N_{v}$ decreases as $1 / \Phi$. Then the maximum heights of the peaks are: $J_{c}\left(\Phi_{1}\right)=1$, $J_{c}\left(2 \Phi_{1}\right)=0.5$, and $J_{c}\left(3 \Phi_{1}\right)=0.33$. Note that these values are obtained provided each pinning site can trap only one vortex, which is justified for the chosen radius of the pinning site and for the vortex densities considered. In addition, there are weak wide maxima corresponding to $\Phi_{1} / 2$ and other "subharmonics", i.e. $\Phi_{i}+\Phi_{1} / 2$ (Fig. 1a), where $i$ is an integer. For very small values of $\Phi_{1}$, the vortex density is very low, and vortices almost do not interact with each other. As a result, in the ground state they all become trapped by pinning sites, and $J_{c}$ is maximal for small $\Phi_{1}$.

For smaller radii of the pinning sites, $r_{p}=0.04 \lambda$ (Fig. 1b), the $J_{c}(\Phi)$ peaks corresponding to matching fields become sharper because for smaller values of $r_{p}$, it is more difficult to fulfill the commensurability conditions. Any features around the main peaks, including subharmonics, are suppressed. Note also a "parity effect" takes place in this case: since the number of pinning sites per cell is odd $\left(N_{p}=25\right.$ in Fig. 1), therefore $J_{c}(\Phi)$ peaks are suppressed for $\Phi_{1} / 2$ and for even values of $i$ in the sequence $\Phi_{i}+\Phi_{1} / 2$.

\section{PINNING OF VORTICES BY A 1D QUASIPERIODIC CHAIN OF PINNING SITES: GRADUAL EVOLUTION FROM A PERIODIC TO A QUASIPERIODIC CHAIN}

Let us consider a QP chain of pinning sites with spacings between pinning sites given by $a_{L}$ (long) and $a_{S}$ (short). The long and short segments alternate according to the Fibonacci rules forming a Fibonacci sequence. The number of pinning sites per cell coincides with the number of elements of this sequence per unit cell. It is natural to take a chain with a number of elements equal to one of the successive Fibonacci numbers as a 1D cell, although in principle it could be of any length. We impose periodic boundary conditions at the ends of the cell.

The larger cell we take, the closer we are to describing a truly QP structure. However, it turns out, that even a finite part of a QP system (1D chain or 2D QP lattice) provides us with reliable information concerning 


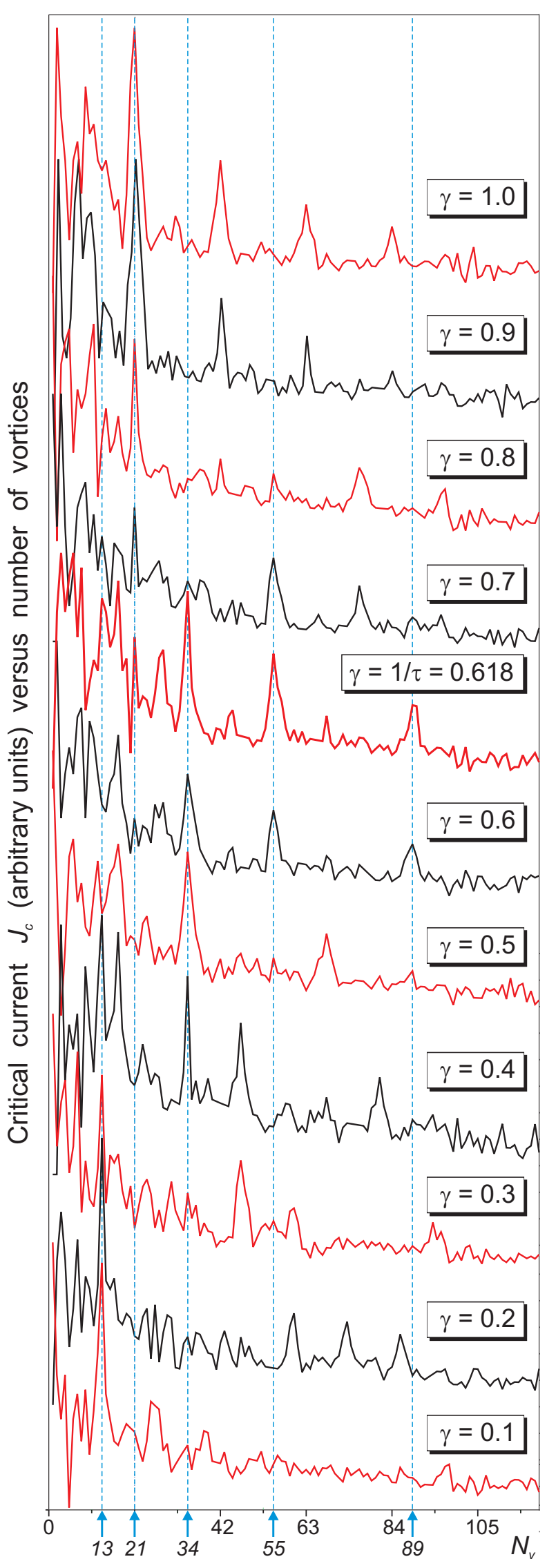

FIG. 2: (Color) Critical depinning current $J_{c}$, as a function of the applied magnetic flux (for convenience, shown as a function of the number of vortices, $\left.N_{v} \sim \Phi\right)$, in a $1 \mathrm{D}$ QP chain of pinning sites with $f_{p} / f_{0}=1, r_{p}=0.1 \lambda$, for chains characterized by different ratios of the lengths of short $a_{S}$ to long $a_{L}$ spacings, $\gamma=a_{S} / a_{L}$. For small deviations of the chain from a periodic chain $(\gamma=0.9$ to 1$)$, commensurability peaks are similar to those shown in Fig. 1. For intermediate values of $\gamma(\gamma=0.2$ to 0.8$)$, peaks are determined by a sequence of harmonics of numbers of long and short periods of the chain, which includes the sequence of successive Fibonacci numbers, most pronounced for $\gamma=1 / \tau \approx 0.618$, where $\tau=(1+\sqrt{5}) / 2 \approx 1.618$ is the golden mean. For very small $\gamma$ (e.g., $\gamma=0.1$, the QP chain effectively becomes periodic but with the number of pinning sites equal to the number of long periods in the chain.

properties of the whole system. This is based on the structural self-similarity of QP systems. These properties are studied here for the critical depinning current $J_{c}$ and have also been demonstrated for other physical phenomena 14, 15, 17, 18, 19, 20, 28].

Figure 2 shows the evolution of $J_{c}$ as a function of the number of vortices, $N_{v} \sim \Phi$, for various values of the parameter $\gamma$. The top curve represents the limiting case of a periodic chain $(\gamma=1.0)$ with typical peak structure discussed above (Fig. 1). The chain contains $N_{p}=21$ pinning sites. As a result, commensurability peaks appear at $N_{v}=21,42,63$, etc., i.e. multiples of 21 . A small QP distortion of the chain does not appreciably affect the peak structure of the function $J_{c}\left(N_{v}\right)(\gamma=0.9)$. When the deviation of the factor $\gamma$ from unity becomes larger $(\gamma=0.8,0.7)$, commensurability peaks for $N_{v}=42,63$, and other multiples of 21 decrease in magnitude. At the same time, new peaks appear at $N_{v}=55,76(=55+21)$, $97(=76+21)$ for $\gamma=0.8$. Then, with further decrease of $\gamma(\gamma=0.7)$, these peaks remain $\left(N_{v}=55\right.$ even grows in magnitude), and a new peak at $N_{v}=34$ arises.

For the golden mean-related value of $\gamma=1 / \tau \approx 0.618$, we obtain a set of peaks, which are "harmonics" of the numbers of long and short periods of the chain (or reciprocal lengths $a_{L}$ and $a_{S}$ ), i.e.

$$
x_{\text {peaks, } i}^{Q P}=A_{i} N_{L}+B_{i} N_{S}=\frac{A_{i}^{\prime}}{a_{S}}+\frac{B_{i}^{\prime}}{a_{L}},
$$

where $N_{L}$ and $N_{S}$ are the numbers of long and short elements, respectively; $A_{i}\left(A_{i}^{\prime}\right)$ and $B_{i}\left(B_{i}^{\prime}\right)$ are generally (positive or negative) multiples or divisors of $N_{L}$ and $N_{S}$; the upper index "QP" denotes "quasiperiodic". It is easy to see that this set includes as a subset the sequence of successive Fibonacci numbers. In particular, the following well-resolved peaks of the function $J_{c}\left(N_{v}\right)$ appear for $\gamma=1 / \tau \approx 0.618: N_{v}=13\left(=N_{L}=13,13\right.$ is a Fibonacci number $(\mathrm{FN})) ; N_{v}=17\left(=\left(2 N_{L}+N_{S}\right) / 2\right.$, where $\left(2 N_{L}+N_{S}\right)=34$ is a FN); $N_{v}=21\left(=N_{p}=N_{L}+N S\right.$, 21 is a FN $) ; N_{v} \approx 27-28\left(=\left(3 N_{L}+2 N_{S}\right) / 2\right.$, where $\left(3 N_{L}+2 N_{S}\right)=55$ is a FN $) ; N_{v}=34\left(2 N_{L}+N_{S}\right)$; $N_{v} \approx 44-45\left(=\left(5 N_{L}+3 N_{S}\right) / 2\right.$, where $\left(5 N_{L}+3 N_{S}\right)=89$ 
FIG. 3: (Color) (a) The critical depinning current $J_{c}$, as a function of the number of vortices, $N_{v} \sim \Phi$, for different $1 \mathrm{D}$ QP chains, $N_{p}=21$ (red bottom line), $N_{p}=34$ (blue line), $N_{p}=55$ (green line), and $N_{p}=89$ (dark blue top line), and the same $\gamma=a_{S} / a_{L}=1 / \tau$. The parameters used here are: $f_{p} / f_{0}=1.0$ and $r_{p}=0.1 \lambda$. Independently of the length of the chain, the peaks for all of the curves include as a subset the sequence of successive Fibonacci numbers (indicated by blue arrows in the horizontal axis) and their sub-harmonics. (b) $J_{c}\left(N_{v}\right)$ for a long chain $N_{p}=144$ and the same $\gamma=$ $1 / \tau$. Notice that now $N_{v}$ is much larger than in (a). (c) The function $J_{c}\left(\Phi / \Phi_{1}\right)$ for the same set of $1 \mathrm{D}$ chains (using the same colors), normalized by the number of pinning sites for each chain. The curves for chains with different $N_{p}$ 's display the same sets of peaks, namely, at $\Phi / \Phi_{1}=1$ (first matching field) and $\Phi / \Phi_{1}=0.5$, as well as at the golden-mean-related values: $\Phi / \Phi_{1}=\tau / 2, \Phi / \Phi_{1}=(\tau+1) / 2=\tau^{2} / 2, \Phi / \Phi_{1}=\tau$, $\Phi / \Phi_{1}=\left(\tau+\tau^{2}\right) / 2=\tau^{3} / 2, \Phi / \Phi_{1}=\tau^{2}=\tau+1, \Phi / \Phi_{1}=\tau^{2}+1$. Therefore, similar sets of peaks are obtained for both cases: for the curves plotted in the same scale (a), (b); for the curves plotted in individual scales, i.e., normalized on the number of pinning sites in the chain (c). This behavior demonstrates the self-similarity of $J_{c}(\Phi)$.

is a $\mathrm{FN}) ; N_{v}=55\left(=\left(3 N_{L}+2 N_{S}\right), 55\right.$ is a $\left.\mathrm{FN}\right) ; N_{v}=68$ $\left(=\left(4 N_{L}+2 N_{S}\right)\right) ; N_{v}=89\left(=\left(5 N_{L}+3 N_{S}\right), 89\right.$ is a FN). In summary, the most pronounced peaks are at: $N_{v}=13,17,21,34,55,89$, which (except the point $N_{v}=17=34 / 2$ ) form a sequence of successive FNs.

The above QP peaks only slightly degrade at $\gamma=0.6$. However, when the length of the long segment $a_{L}$ becomes twice the length of the short one $a_{S}$, i.e. $\gamma=0.5$, sharp commensurability peaks appear which are related to the small segment of the chain with length $a_{S}=a_{L} / 2$. Namely, we obtain peaks at $N_{v}=34 \sim\left(2 N_{L}+N_{S}\right)$ and at other values of $N_{v}$ which are sub-harmonics of $2 N_{L}+N_{S}: N_{v}=17,34,68$. Other peaks, in particular, those related to the Fibonacci sequence, are much less pronounced for $\gamma=0.5$. For $\gamma=0.4$, the peaks are at: $N_{v}=13,17,34,47\left(=3 N_{L}+N_{S}\right), 68$, and 81 . A very strong (recall that the maximum amplitude of the peak is $\sim 1 / N_{v}$ ) peak at $N_{v}=81$ is a "resonance" peak corresponding to the ratio of $B_{i} / A_{i}=2 / 5=\gamma$, i.e. $5 N_{L}+2 N_{S}=81$. When $\gamma=0.3$, the "resonance" ratio $B_{i} / A_{i}=0.3$, therefore, a strong peak appears for the "nearest to 0.3 " value $B_{i} / A_{i}=0.33: 3 N_{L}+N_{S}=47$, and also for $6 N_{L}+2 N_{S}=94$. Another "close to 0.3 " value is $B_{i} / A_{i}=0.25$, which is responsible for the peaks at $N_{v}=30\left(2 N_{L}+N_{S} / 2\right)$ and $N_{v}=60\left(4 N_{L}+N_{S}\right)$. Also, peaks at $N_{v}=13,17$ and 55 are present. The "resonant" peak for $\gamma=0.2$ (i.e., for the ratio $\gamma=B_{i} / A_{i}=0.2$ ), appears at $N_{v}=73\left(=5 N_{L}+N_{S}\right)$. The closest neighboring peaks are at $N_{v}=60\left(=4 N_{L}+N_{S}\right)$ and $N_{v}=86$ $\left(=6 N_{L}+N_{S}\right)$, [also: $\left.N_{v}=43\left(=3 N_{L}+N_{S} / 2\right)\right]$, characterized by ratios $B_{i} / A_{i}=0.25$ and $B_{i} / A_{i}=0.17$, correspondingly. Finally, for $\gamma=0.1$ we arrive at the situation when we have an almost periodic chain but with a period $a$ different from that for $\gamma=1.0: a^{\prime} \approx a_{L}$ since $a_{S} \ll a_{L}$.
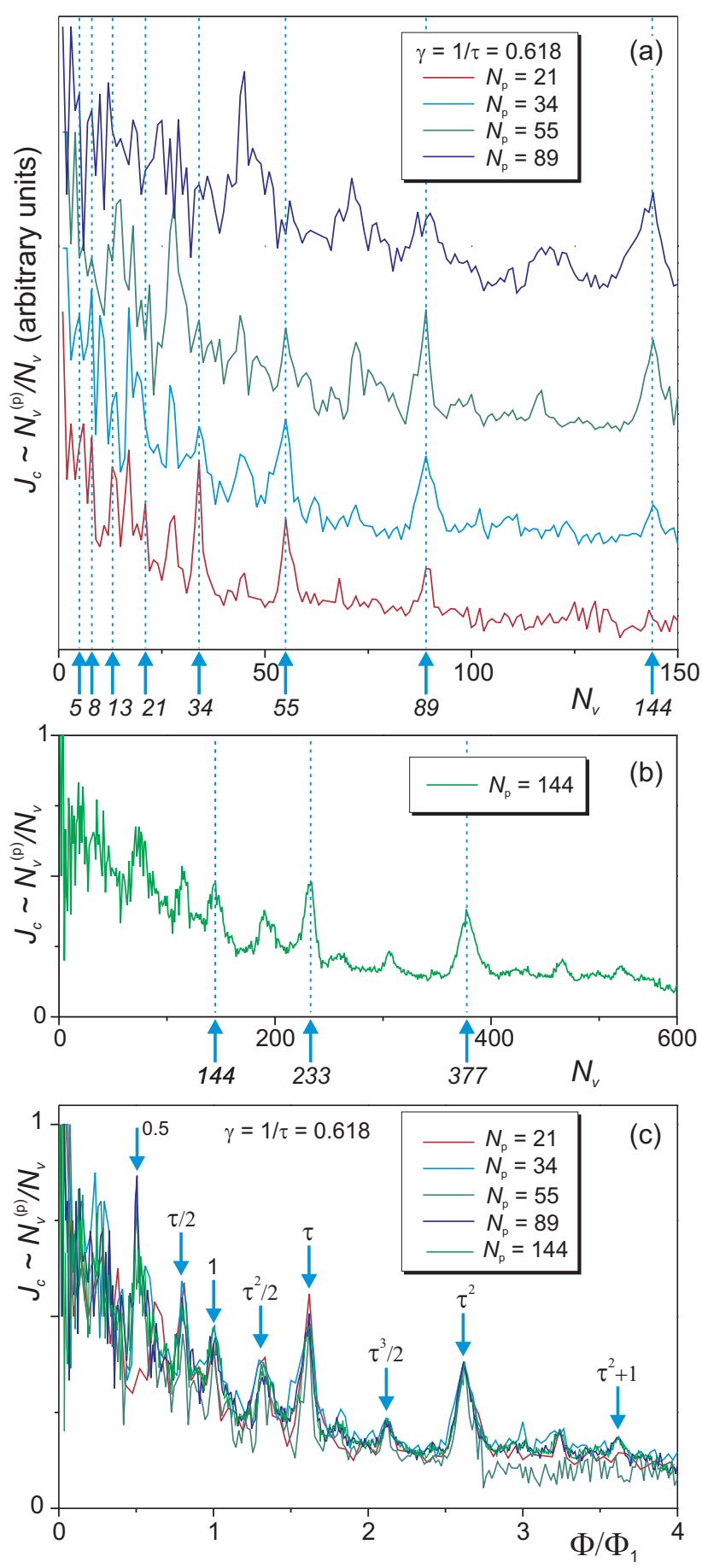

The number of pinning sites becomes $N_{p}=N_{L}=13$, and we obtain commensurability peaks at the positions which are multiples of $N_{p}=13$, i.e., at $N_{v}=13,26,39$, 52 , which is typical for periodic chains of pinning sites.

In order to demonstrate that the above analysis is general and reveals the QP features independently of the length of a specific chain of pinning sites, let us compare results from the calculation of $J_{c}\left(N_{v}\right)$ for different chains. In Fig. 3a, the function $J_{c}\left(N_{v}\right)$ is shown for four different 1D QP chains, $N_{p}=21, N_{p}=34, N_{p}=55$, and $N_{p}=89$, and the same $\gamma=1 / \tau$. Figure 3a clearly shows that the 
positions of the main peaks in $J_{c}$, i.e. those corresponding to a Fibonacci sequence, and other peaks whose positions are described by Eq. (12), to a significant extent, do not depend on the length of the chain. The peaks shown in Fig. 3 form a Fibonacci sequence: $N_{v}=13,21,34,55,89$, 144, and other "harmonics": $N_{v}=17,27-28(=55 / 2)$, 44-45 (=89/2), etc. At the same time, longer chains allow to better reveal peaks for larger Fibonacci numbers. Thus, for chains with $N_{p}=144$ (Fig. $3 \mathrm{~b}$ ) peaks at the next Fibonacci numbers are pronounced: $N_{v}=144,233$, 377.

While the curves for different chains are plotted in Fig. 3a in the same scale, Fig. 3c shows these curves in individual scales. Namely, we rescale each $J_{c}$ by normalizing each $J_{c}$ by the number of pinning sites in each curve. Thus, $\Phi_{1}$ corresponds to $N_{v}=21$ for the chain with $N_{p}=21$, to $N_{v}=34$ for the chain with $N_{p}=34$, etc. After this rescaling, the $J_{c}$ curves approximately follow each other and have pronounced peaks for the goldenmean-related values of $\Phi / \Phi_{1}$, as shown in Fig. 3c. For example, $\Phi / \Phi_{1}=\tau$ corresponds to $N_{v}=34$ for the chain with $N_{p}=21$, to $N_{v}=55$ for the chain with $N_{p}=34$, to $N_{v}=89$ for $N_{p}=55$, to $N_{v}=144$ for $N_{p}=89$, and to $N_{v}=233$ for the chain with $N_{p}=144$. Note that these peaks (i.e., corresponding to the golden mean) are most pronounced for each chain in Fig. 3a,b.

Therefore, the same peaks of the function $J_{c}(\Phi)$ for different chains are revealed before and after rescaling. This means that the function $J_{c}(\Phi)$ for the 1D QP chain is self-similar. Below, we demonstrate the revealed selfsimilarity effect in a reciprocal $k$-space.

\section{A. Fourier-transform of the vortex distribution function on a $1 \mathrm{D}$ periodic chain of pinning sites: Self-similarity effect}

As we established above, the revealed QP features (e.g., peaks of the function $J_{c}\left(\Phi \sim N_{v}\right)$ ) (i) are independent of the length of the chain and (ii) the longer chain we take, the more details (e.g., "subharmonics") of QP features can be observed.

This result is related to an important property of QP systems, self-similarity, which could be better understood by analyzing the Fourier-transform of the distribution function of the system of vortices pinned on a QP array.

In $k$-space, the distribution function $F_{v}^{(p)}(q)$ of a system of $N_{v}^{(p)}$ pinned vortices can be represented as the inverse Fourier-transform of the 1D distribution function $F_{v}^{(p)}(n)$ of the vortices in real space:

$$
F_{v}^{(p)}(q)=\frac{1}{N_{v}^{(p)}} \sum_{n=1}^{N_{v}^{(p)}} F_{v}^{(p)}(n) \exp \left\{-2 \pi i q n / N_{v}^{(p)}\right\} .
$$

Figure 4 shows the Fourier-transform of a system of pinned vortices for a $\gamma$ equal to the inverse golden mean: $1 / \gamma=a_{L} / a_{S}=\tau=(1+\sqrt{5}) / 2$. The plots shown in

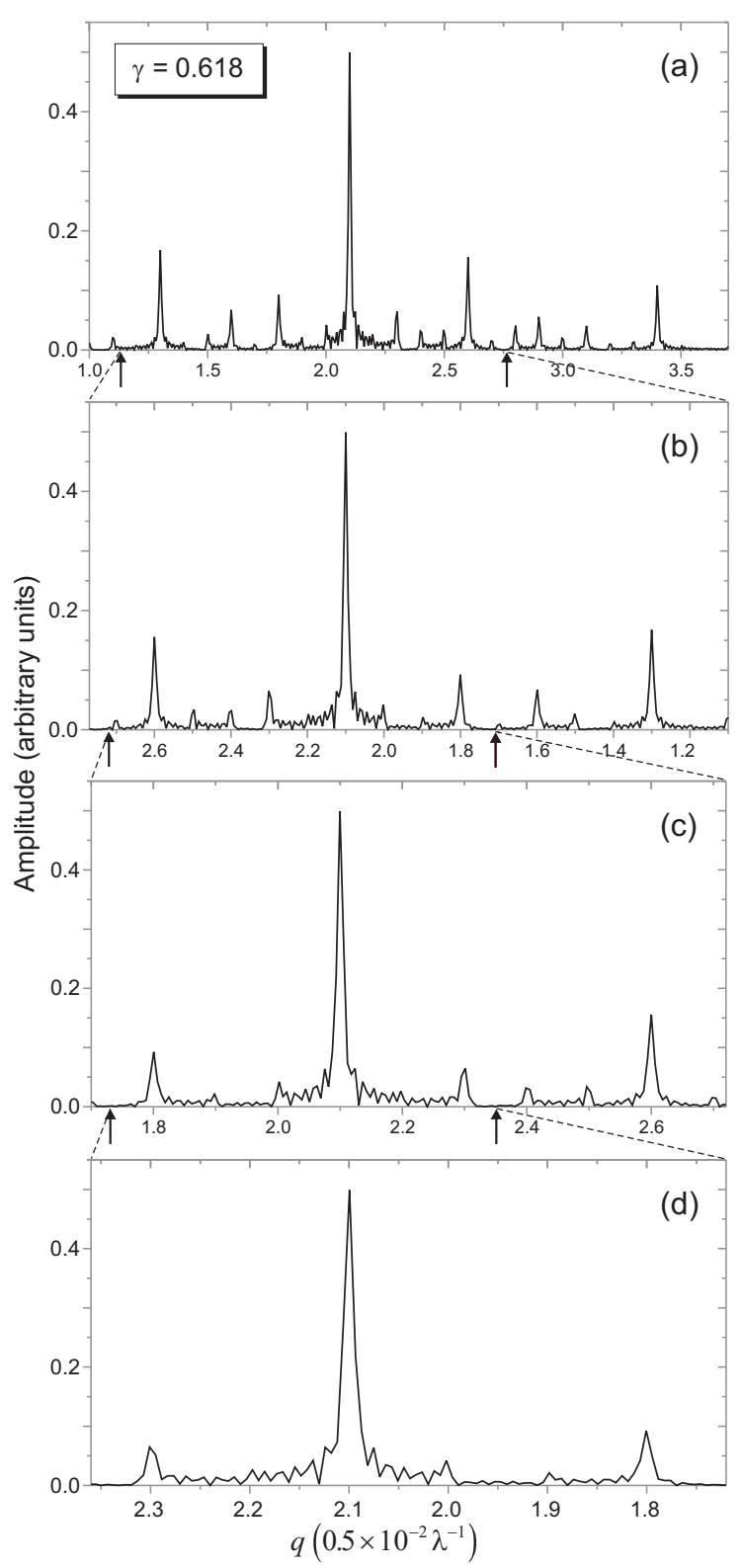

FIG. 4: The self-similar Fourier-transform of the distribution function (vortex density) of the system of $N_{v}=144$ vortices pinned on a QP array, for $\gamma=1 / \tau$. The portion limited by the two arrows in (a) is successively magnified several times and the corresponding results shown in (b), (c) and (d).

Figs. 4a to $4 \mathrm{~d}$ are obtained according to the following rule. The portion of the horizontal axis which is limited by the two arrows in Fig. 4a is rescaled and shown in Fig. 4b. In the same way, the portion limited by the two arrows in Fig. 4b is rescaled and shown in Fig. 4c. Fig. 4d is obtained following the same procedure. Note that each subsequent scaling is accompanied by flipping the direction of the $q$-axis to the opposite direction. A similar property is clear from the experimental diffraction patterns of quasicrystals 11]. The pentagons of Bragg 


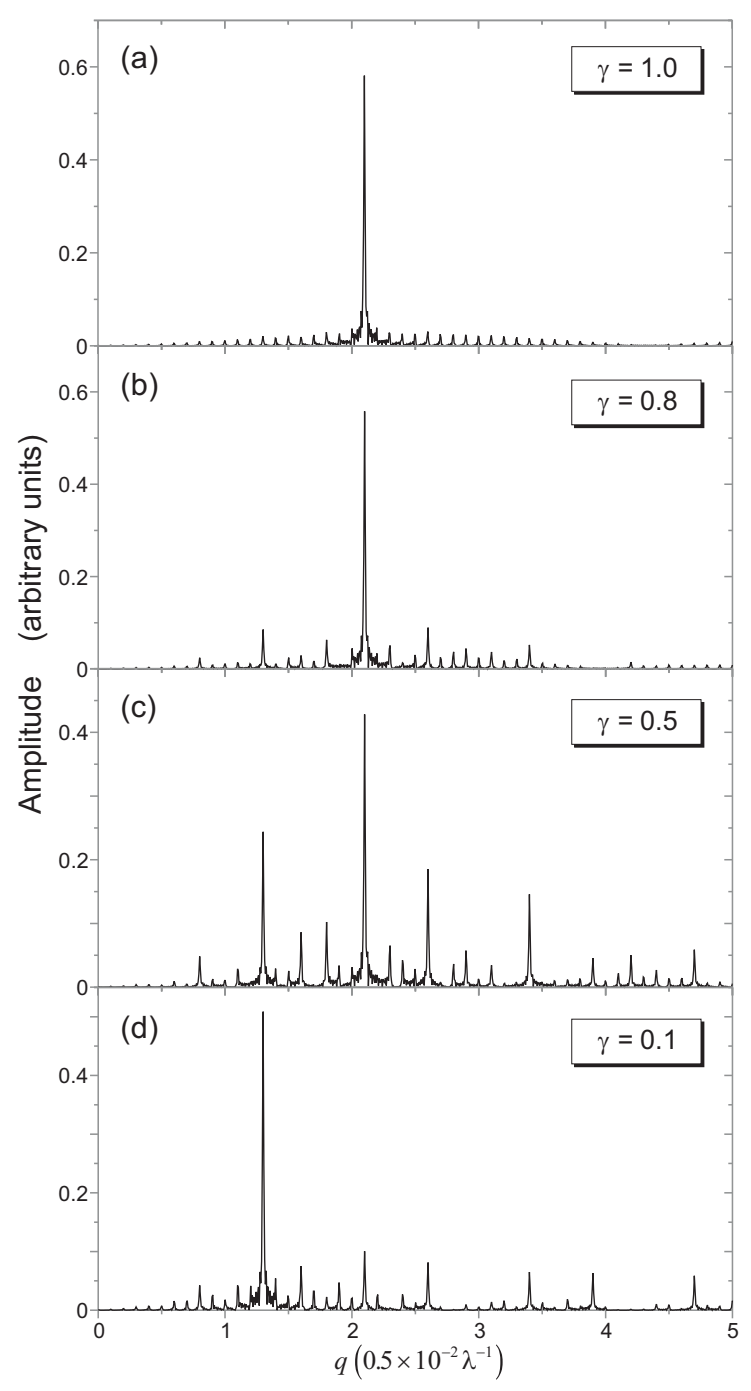

FIG. 5: Fourier-transform of the distribution function of the vortices $\left(N_{v}=144\right)$ interacting with a QP array of pinning sites, for different values of $\gamma=1.0$ (a), 0.8 (b), 0.5 (c), 0.1 (d). The value $\gamma=1$ corresponds to the limit of a periodic chain. Varying $\gamma$ from one, we introduce quasiperiodicity (the most pronounced for $\gamma=1 / \tau \approx 0.618$ ) in the chain. The $\gamma=1$ case recovers the periodic limit (with another period). The main central sharp peak, corresponding to the periodic chain used for (a), continuously transforms - through the set of selfsimilar patterns ((b) and (c)) corresponding to QP chains (see Fig. 4) - to another peak (d) produced by a periodic chain with the number of sites equal to the number of long segments of the initial periodic chain with $\gamma=1.0$ shown in (a).

peaks have smaller pentagons inside them, which are inverted. As seen in Fig. 4, each subsequent subdivision leads to a subset of peaks similar to the entire set of peaks.

This analysis clearly demonstrates the self-similarity of the distribution function of the vortices pinned on a QP 1D array of pinning sites. Similarly to the observed be- havior of the function $J_{c}(\Phi)$ when increasing the length of the chain of pinning sites, the Fourier-transform of the distribution function of the vortices pinned on a $\mathrm{QP}$ array reproduce its main features (peaks) in a self-similar way, when increasing the range in $k$-space, and simultaneously acquires a more elaborate structure with smaller self-similar peaks.

As we discussed above, the main commensurability peaks evolve from a perfectly periodic set of the type $\Phi_{i}=m \Phi_{1}$, where $m>1$ is a positive integer, (through the set of QP peaks defined by Eq. (12)), to another set of periodic peaks $\Phi_{i}^{\prime}=m \Phi_{1}^{\prime}$, when the "quasiperiodicity parameter" $\gamma \equiv a_{S} / a_{L}$ is gradually tuned between the values $\gamma=1.0$ and $\gamma=0$ (see Fig. 2). These limits $(\gamma=1.0$ and $\gamma=0)$ correspond to periodic chains.

Fig. 5 illustrates the corresponding evolution of the Fourier-transform of the distribution function of vortices pinned on a $1 \mathrm{D}$ QP array of pinning sites.

For $\gamma=1$ (Fig. 5a), there is a single sharp peak (accompanied by negligibly small satellites) corresponding to a periodic chain. For smaller $\gamma$ 's (e.g., $\gamma=0.8$ (Fig. 5b), $\gamma=0.8$ (Fig. 5c), or $\gamma=0.618$ (Fig. 4a)), a set of satellite peaks appears around the main peak. Simultaneously, the intensity of this peak decreases giving rise to another main peak for smaller value of $q$ (Fig. $5 \mathrm{~d}$ ), which corresponds to a periodic chain with a larger period.

\section{PINNING OF VORTICES BY 2D QUASIPERIODIC PINNING ARRAYS}

In the previous section we studied the pinning of vortices by 1D QP chains of pinning sites. In particular, we showed how the quasiperiodicity manifests itself in the critical depinning current, $J_{c} \sim N_{v}^{(p)} / N_{v}$, when increasing the applied magnetic flux, $\Phi \sim N_{v}$. We found that the positions of the peaks of the function $J_{c}(\Phi)$ are governed by "harmonics" of long and short periods of the QP chain of pinning sites. Independently of the length of the chain (for $N_{p} \geq 21$ ), the peaks form a QP sequence including the Fibonacci sequence as a fundamental subset. This self-similarity effect is clearly displayed in the Fourier-transform of the distribution function of the vortices on a 1D QP array of pinning centers. The evolution of QP peaks, when gradually changing the "quasiperiodicity" parameter $\gamma$, has revealed a continuous transition from a QP chain - through the set of QP states (most pronounced for $\gamma=1 / \tau \approx 0.618$ ) — to another periodic chain, $\gamma=0$, with a longer period. This phenomenon has been studied both in real space and in reciprocal $k$-space.

In the present section and in the next sections, we analyze vortex pinning by $2 \mathrm{D} \mathrm{QP}$ arrays; in particular, by an array of pinning sites placed in the nodes of a fivefold Penrose lattice. Before tackling the Penrose-lattice pinning array, let us start with a simplified system which one can call "2D-quasiperiodic" (2DQP) since it is a $2 \mathrm{D}$ system periodic in one direction ( $x$-direction) and $\mathrm{QP}$ 
in the other direction ( $y$-direction).

\section{A. 2D-quasiperiodic triangular lattice of pinning sites}

In an infinitely long one-dimensional homogeneous superconductor without any pinning centers, vortices obviously are equidistantly distributed, forming a periodic chain. Similarly, as it is well-known, in a threedimensional superconductor (or in quasi-two-dimensional slabs or films), vortices organize themselves in a periodic triangular lattice shown schematically in Fig. 6a. If we keep the lattice undistorted along the $x$-direction and introduce a quasiperiodic deformation along the $y$ direction, similarly to the case of a 1D QP chain, we obtain a 2QP triangular lattice as shown in Figs. 6b,c. The "quasiperiodicity" parameter $\gamma_{y}$ for this 2DQP lattice is defined as the ratio of the short to long periods, $a_{L}$ to $a_{S}$ (see Fig. 6c). The 2DQP triangular arrays of pinning sites are shown for $\gamma_{y}=1.0$ (Fig. 6a), $\gamma_{y}=1 / \tau$, where $\tau=(1+\sqrt{5}) / 2$ (Fig. 6b), $\gamma_{y}=0.5$ (Fig. 6c). The corresponding functions $J_{c}\left(N_{v} \sim \Phi\right)$ are presented in Figs. 6d,e,f for the following pinning parameters: $f_{p} / f_{0}=2.0$, and $r_{p}=0.1 \lambda$.

For the triangular array of pinning sites (Fig. 6a), we obtain a well-resolved main commensurability peak (Fig. 6d), corresponding to the first matching field, at $\Phi=\Phi_{1}$. Note that the vortex lattice is in general incommensurate with a triangular lattice of pinning sites for the second matching field, i.e. when $\Phi=2 \Phi_{1}$ (see, e.g., Ref. 25]). For instance, for the parameters used in our simulations, only each second row of pinning sites is occupied at $\Phi=2 \Phi_{1}$, resulting in a very weak maximum of the function $J_{c}\left(N_{v} \sim \Phi\right)$ at that point; the parameters used $\left(f_{p} / f_{0}=2.0, r_{p}=0.1 \lambda\right)$ are nearly optimal for revealing features of the function $J_{c}(\Phi)$ related to quasiperiodicity.

When tuning $\gamma$ out of the periodic value $\gamma_{y}=1.0$, the main peak decreases in magnitude, and a maximum forms near it at a larger value of $N_{v} \sim \Phi$. These changes are demonstrated in Fig. 6e (the corresponding pinning array is shown in Fig. 6b) for $\gamma_{y}=1 / \tau$.

It should be noted that here the parameter $\gamma_{y}$ has a different meaning, in the case of triangular 2DQP lattices, compared to the case of $\gamma$ for the 1D QP chains considered in the previous section. In a $1 \mathrm{D} \mathrm{QP}$ chain, $\gamma=a_{S} / a_{L}$ is the ratio of distances between pinning sites, whereas in a triangular 2DQP lattice $\gamma_{y}$ defines the ratio of the distances between the rows of pinning sites (see Fig. 6c). It is easy to show that the ratio of distances between the neighboring pinning sites in a triangular 2DQP lattice is

$$
\gamma^{\prime}=\sqrt{\frac{\left(1+\gamma_{y}^{2}\right) a^{2}+12 a^{2}}{\left(1+\gamma_{y}^{2}\right) a^{2}+12 \gamma_{y}^{2} a^{2}}},
$$

where $a$ is the period of the 2DQP triangular lattice along the (periodic) $x$-direction. Thus, for $\gamma_{y}=1 / \tau$, the parameter $\gamma^{\prime}$ defined by Eq. (14) becomes $\gamma^{\prime} \approx 0.7$.

For $\gamma_{y}=0.5\left(\gamma^{\prime} \approx 0.6\right)$, the function $J_{c}\left(N_{v}\right)$ is plotted in Fig. 6f. The main peak is further depressed, while the closest satellite peak becomes more pronounced. In addition, other satellite peaks appear, which are much less pronounced.

\section{TRANSITION FROM A TRIANGULAR TO A QUASIPERIODIC PENROSE-LATTICE ARRAY OF PINNING SITES}

Above, we have revealed some features of the behavior of the critical depinning current $J_{c}$ as a function of the applied magnetic flux $\Phi$ (or as a function of the number of vortices in the system $\left.N_{v} \sim \Phi\right)$. They have been found under the transformation of a triangular lattice to a 2DQP triangular lattice (array) of pinning sites.

Consider now a similar procedure but the final configuration of the transformation from a triangular lattice will be a 2D QP array of pinning sites, namely, an array of pinning sites located at the nodes of a five-fold Penrose lattice. This kind of lattice is representative of a class of 2D QP structures, or quasicrystals, which are referred to as Penrose tilings. These structures possess a local order and a rotational (five- or ten-fold) symmetry, but do not have translational long-range order. Being constructed of a series of building blocks of certain simple shapes combined according to specific local rules, these structures can extend to infinity without any defects [1]. Below, we will discuss in more detail the structure of the Penrose lattice.

The transformation of a triangular lattice to the Penrose one is a rather non-trivial procedure, as distinct from the transformation to a $2 \mathrm{DQP}$ triangular lattice done above when we simply stretched some of the interrow distances and squeezed other ones according to the Fibonacci rules (Eq. (8)) for a one-dimensional QP lattice. In order to find intermediate configurations between the triangular lattice and the Penrose lattice, we employ the following approach. First, we place non-interacting vortices at the positions coinciding with the nodes of the Penrose lattice (these can be considered as pinning sites for vortices, which right afterwards are "switched off"). Then we let the vortices freely relax undergoing the vortex-vortex interaction force at low temperatures (and no pinning force). The vortices relax to their ground state, which is a triangular lattice. During the relaxation process, we do a series of "snapshots", recording the coordinates of the vortex configurations at different times. The sets of coordinates obtained are then used as coordinates of pinning sites. We arrange these sets in antichronological order to model a continuous transition of the pinning array from its initial configuration, a triangular lattice, to its final configuration, a Penrose 

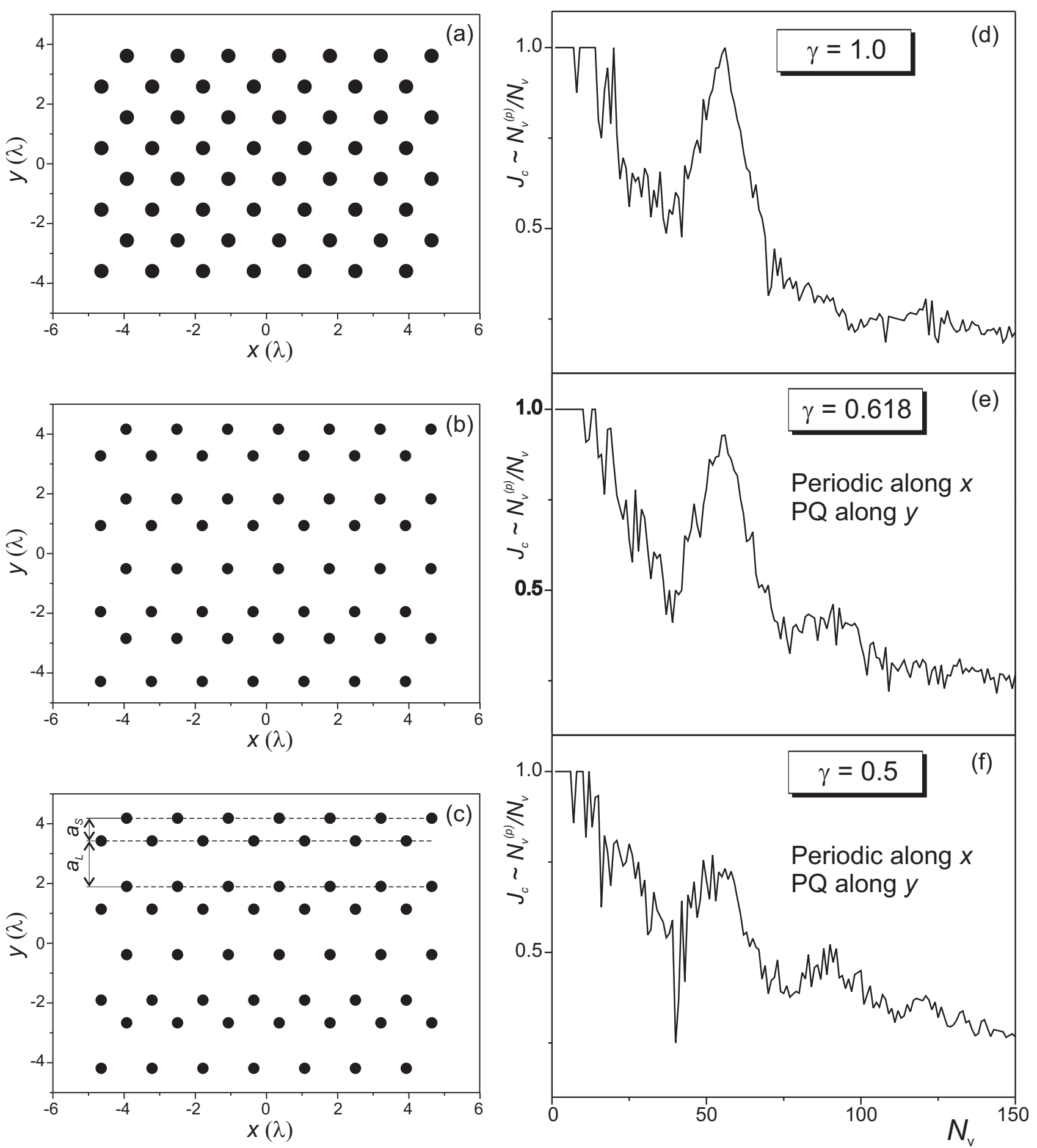

FIG. 6: The spatial distribution of pinning sites for a triangular lattice (i.e., periodic) (a), and 2D-quasiperiodic (2DQP) triangular, i.e. periodic along one direction (the $x$-direction) and QP along the other one (the $y$-direction), (b) and (c). The parameter $\gamma_{y}$ is defined as a ratio of short $\left(a_{S}\right)$ to long $\left(a_{L}\right)$ periods in the $y$-direction, as shown in (c). The values of the parameter $\gamma_{y}$ are: $\gamma_{y}=1.0$ (a), $\gamma_{y}=1 / \tau$, where $\tau=(1+\sqrt{5}) / 2$ (golden mean) (b), $\gamma_{y}=0.5$ (c). The critical depinning current $J_{c}$, as a function of the number of vortices, $N_{v} \sim \Phi$, for triangular (d) and the 2DQP triangular lattices [shown in (b) and (c)] (e) and (f), correspondingly, for $f_{p} / f_{0}=2.0, r_{p}=0.1 \lambda$. 
FIG. 7: (This figure is available in "png" format: "Penrose Fig 7.png"; color) Left column: Transformation of a triangular lattice of pinning sites to a five-fold Penrose lattice. The distributions of the pinning sites in the triangular lattice (shown by blue solid circles in (a) and by blue open circles in (b), (c), and (d), for comparison). Intermediate configurations (shown by red solid circles in (b) and (c)) between the triangular (a) and the Penrose lattice (shown by red solid circles in (d)). Right column: The corresponding critical depinning currents, $J_{c}$, as a function of the applied magnetic flux, $\Phi$, for the pinning arrays shown in (a) to (d), respectively: for the triangular lattice (shown by blue solid line in (e) and by blue dashed lines in (f), (g), and (h), for comparison); for the intermediate configurations (shown by red solid lines in (f) and (g)); for the Penrose lattice (shown by the red solid line in (h)). For the Penrose lattice case in (b), the drop in $J_{c}(\Phi)$ is an artifact of the boundary conditions. Namely, the Penrose lattices of pinning sites did not fit the square cell used in the simulations. Thus, the freely-moving vortices near the edges significantly decreased the value of $J_{c}$, especially near $\Phi_{1}$. This problem will be dealt separately in Fig. 14 and in Eq. (16).

lattice.

In Figs. 7a to 7d, four of these configurations of pinning sites are shown. The triangular lattice is presented in Fig. 7a. Two intermediate configurations are shown in Figs. 7b,c. The pinning sites plotted in Fig. $7 \mathrm{~d}$ are located on the vertices of a Penrose lattice. For comparison, a pinning array in the form of a triangular lattice (Fig. 7a) is also presented in Figs. 7b,c,d as open blue circles. The functions $J_{c}(\Phi)$ calculated for the pinning arrays shown in Figs. 7a to d, are plotted, respectively, in Figs. 7e to h. The function $J_{c}(\Phi)$ for the triangular lattice (Fig. 7e) is also plotted, for comparison, in Figs. $7 g, f, h$ as a blue dashed curve.

The main commensurability peak related to the first matching field in a triangular lattice of pinning sites, observed at $\Phi=\Phi_{1}$ (Fig. 7e), turns out to be rather stable with respect to moderate deformations of the lattice (Fig. 7f, see also Fig. 7b). It still has a maximum height in Fig. 7f, although it broadens. However, the depths of the valleys near the peak decreases by about 20 to 30 per cent. Two sharp peaks near $\Phi=\Phi_{1} / 3$ and $\Phi=\Phi_{1} / 6$ (Fig. 7e), related to the commensurability of the longrange order in a triangular lattice, disappear. With further deformation, e.g., for the pinning array shown in Fig. 7c, the main peak still remains but only about 80 per cent of the vortices are pinned in this case. The function $J_{c}(\Phi)$ becomes somewhat smoother, and it does not display any pronounced features (for $\Phi \gtrsim 2 \Phi_{1} / 3$ ) except the main maximum.

The transition to a Penrose-lattice array of pinning sites (Fig. 7d) is accompanied by the appearance of a specific fine structure of the function $J_{c}(\Phi)$. Namely, two well-resolved features on the broad main maximum (Fig. 7h) are the most pronounced ones. Other, less pronounced, features will be discussed below for larger Penrose-lattice arrays. For large arrays, the function $J_{c}(\Phi)$ is much less affected by fluctuations related to the entrance of each single vortex in the system, which are significant for the small-size array shown in Fig. $7 \mathrm{~d}$ $\left(N_{p}=56\right)$. This small-size array is used here just as an illustration, for studying the transition form a periodic (triangular) to a QP (Penrose lattice) pinning site array. However, studying even a relatively small piece of a QP structure provides some useful information about properties of the whole system based on the self-similarity of the lattice, which was revealed for 1D QP chains in the previous section, and which will be demonstrated below for $2 \mathrm{D} \mathrm{QP}$ structures.

The Penrose and the 2DQP triangular lattice (Fig. 6f) both have an important similar feature: their $J_{c}(\Phi)$ has two nearby maxima. Thus, our previous analysis based on several alternative ways to continuously deform a periodic lattice to a $\mathrm{QP}$ one shows that the features shown are hallmarks of QP pinning arrays.

In the next section, the origin of the features observed will be explained on the basis of a detailed analysis of the structure and of the building blocks forming a five-fold Penrose lattice. Other, less pronounced, features will also be discussed. Some of them will be found in larger arrays in our numerical simulations.

\section{ANALYSIS OF THE FINE STRUCTURES OF THE FUNCTION $J_{c}(\Phi)$ IN A QUASIPERIODIC PENROSE-LATTICE ARRAY OF PINNING SITES}

The structure of a five-fold Penrose lattice is shown in Fig. 8. As an illustration, a five-fold symmetric fragment which consists of 46 points (nodes of the lattice) is presented (Fig. 8a). According to specific rules, the points are connected by lines in order to display the structure of the Penrose lattice (compare, e.g., to Fig. 7d). The elemental building blocks are rhombuses with equal sides $a$ snd angles which are multiples of $\theta=36^{\circ}$. There are rhombuses of two kinds forming the Penrose lattice (Fig. 8b): (i) those having angles $2 \theta$ and $3 \theta$ (so called "thick"; they are empty in Fig. 8), and (ii) rhombuses with angles $\theta$ and $4 \theta$ (so called "thin"; they are colored in orange in Fig. 8).

Let us analyze the structure of the Penrose lattice from the point of view ot its pinning properties, when pinning sites are placed in the vertices of the lattice. In particular, we are interested whether any specific matching effects can exist in this system between the pinning lattice and the interacting vortices, which define the critical depinning current at different values of the applied magnetic field (i.e., the function $J_{c}(\Phi)$ ).

On the one hand, QP (quasicrystalline) patterns are intrinsically incommensurate with the flux lattice for any value of the magnetic field [16, 17, 18, 19], therefore, in contrast to periodic (e.g., triangular or square) pinning 


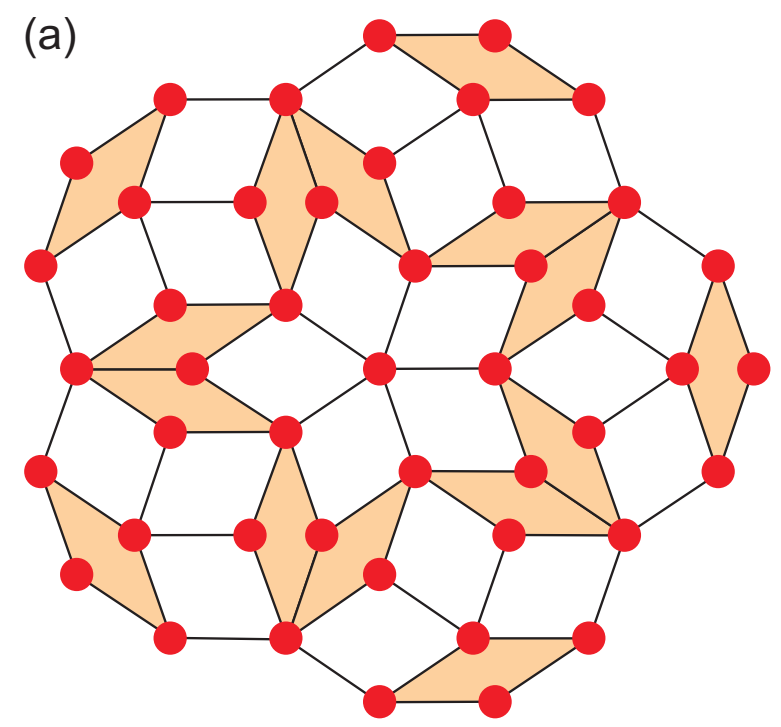

(b)

\section{"Thick" rhombus "Thin" rhombus}

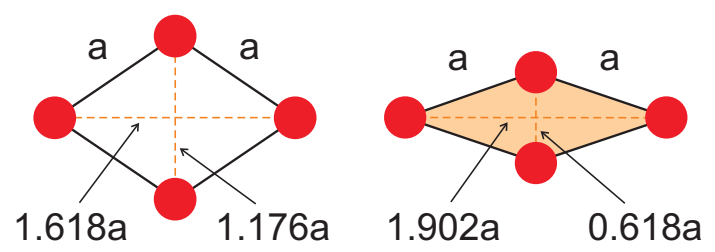

FIG. 8: (Color) The structure of a five-fold Penrose lattice (a). The elemental building blocks are rhombuses with equal sides $a$ and angles which are multiples of $\theta=36^{\circ}$. There are rhombuses of two kinds: those having angles $2 \theta$ and $3 \theta$ (so called "thick"), and rhombuses with angles $\theta$ and $4 \theta$ (so called "thin") (b). The distances between the nodes, i.e. the lengths of the diagonals of the rhombuses are: $1.176 a$ (the short diagonal of a thick rhombus); $(1+\sqrt{5}) a / 2=\tau a \approx 1.618 a$ (the long diagonal of a thick rhombus); $[(1+\sqrt{5}) / 2-1] a=(\tau-1) a \approx$ $0.618 a$, (the short diagonal of a thin rhombus); $1.902 a$ (the short diagonal of a thin rhombus) (b).

arrays, one might a priori assume a lack of sharp peaks in $J_{c}(\Phi)$ for QP arrays of pinning sites.

On the other hand, the existence of many periods in the Penrose lattice can lead to a hierarchy of matching effects for certain values of the applied magnetic field, resulting in strikingly-broad shapes for $J_{c}(\Phi)$.

In order to match the vortex lattice on an entire QP pinning array, the specific geometry of the elements which form the QP lattice is important as well as their arrangement, as distinct from the flux quantization effects and superconductor-to-normal phase boundaries for which the areas of the elements only plays a role [17, 18, 19]. As mentioned above, a five-fold Penrose lattice is constructed of building blocks, or rhombuses, of two kinds. While the sides of the rhombuses are equal (denoted by $a$ ), the distances between the nodes (where we place pin- ning sites) are not equal (which is problematic for vortices). The lengths of the diagonals of the rhombuses are as follows (Fig. 8b): $1.176 a$ (the short diagonal of a thick rhombus); $(1+\sqrt{5}) a / 2=\tau a \approx 1.618 a$, where $\tau$ is the golden mean (the long diagonal of a thick rhombus); $[(1+\sqrt{5}) / 2-1] a=(\tau-1) a \approx 0.618 a$, (the short diagonal of a thin rhombus); $1.902 a$ (the short diagonal of a thin rhombus).

Based on this hierarchy of distances, we can predict matching effects (and corresponding features of the function $J_{c}(\Phi)$ ) for the Penrose-lattice pinning array.

First, we can expect that there is a "first matching field" (let us denote the corresponding flux as $\Phi_{1}$ ) when each pinning site is occupied by a vortex. Although sides of all the rhombuses are equal to each other similarly to that in a periodic lattice, nevertheless this matching effect is not expected to be accompanied by a sharp peak. Instead, it is a broad maximum since it involves three kinds of local "commensurability" effects of the flux lattice: with the rhombus side $a$; with the short diagonal of a thick rhombus, $1.176 a$, which is close to $a$; and with the short diagonal of a thin rhombus, which is the golden mean times $a, 0.618 a$ (see Fig. 8b).

It should be noted that this kind of matching assumes that a vortex lattice is rather weak, i.e. the effect can be more or less pronounced depending on the specific relations between the vortex-vortex interaction constant and the strength of the pinning sites as well as on the distance between pinning sites and their radius. Assuming that the vortex-vortex interaction constant is a material parameter, all others can be adjustable parameters in experiments with artificially-created QP pinning arrays. For instance, the pinning parameters can be "adjusted" by using as pinning centers antidots, i.e. microholes of different radii "drilled" in a superconductor film [1, 2, [6], or blind antidots [7] of different depths and radii.

Further, we can deduce that next to the above "main" matching flux there is another matching related with the filling of all the pinning sites in the vertices of thick rhombuses and only three out of four of the pinning sites in the vertices of thin rhombuses, i.e., one of the pinning sites in the vertices of thin rhombuses is empty. For this value of the flux, which is lower than $\Phi_{1}$, matching conditions are fulfilled for two close distances, $a$ (the side of a rhombus) and $1.176 a$ (the short diagonal of a thick rhombus) but are not fulfilled for the short diagonal, $a / \tau$, of the thin rhombus.

Therefore, this QP feature is related to the golden mean value, although not in such a direct way as in the case of a 1D QP pinning array. This 2D QP matching results in a very wide maximum of the function $J_{c}(\Phi)$. The position of this broad maximum, i.e., the specific value of $\Phi$ (denoted here by $\Phi_{\text {vacancy/thin }} \equiv \Phi_{\mathrm{v} / \mathrm{t}}=0.757 \Phi_{1}$ ) could be found as follows. The ratio of the numbers of thick and thin rhombuses is determined by the Fibonacci numbers and in the limit of large pinning arrays, $N_{p} \rightarrow \infty$ this ratio tends to the golden mean. The number of unoccupied pinning sites is governed by the num- 
FIG. 9: (This figure is available in "png" format: "Penrose Fig 9.png"; color) The critical depinning current $J_{c}$ as a function of the applied magnetic flux, $\Phi \sim N_{v}$, for an array of pinning sites placed at the nodes of a five-fold Penrose lattice (for a part of the lattice which contains $N_{p}=46$ pinning sites) (a). The distributions of vortices (shown by green dots) pinned on the Penrose-lattice pinning site array (pinning sites are shown by red open circles connected by orange solid lines used in order to show the Penrose lattice structure, i.e., thick and thin rhombuses), for specific values of the applied magnetic flux: (b) $\Phi=\Phi_{\text {vacancy } / \text { thin }} \equiv \Phi_{\mathrm{v} / \mathrm{t}}=0.757 \Phi_{1}$, vortices occupy all the pinning sites except those in one of the two vertices (connected by the short diagonal) of each thin rhombus, each single and each pair of double thin rhombuses contain one unoccupied pinning site at the matching field $\Phi_{\mathrm{v} / \mathrm{t}}$; (c) $\Phi=\Phi_{1}$, the number of vortices $N_{v}$ coincides with the number of pinning sites $N_{p}$, and almost all the vortices are pinned (because of using a square simulation cell, some of the vortices are always "interstitial" but allow to keep the average vortex density in the entire simulation cell; due to these additional vortices, the value of the function $J_{c}(\Phi)$ effectively reduces by a "filling" factor $\eta=A_{P} / A \approx 0.575$, where $A_{P}$ and $A$ are the areas of the Penrose-lattice "sample" and of the simulation region; (d) $\Phi=\Phi_{\text {interstitial } / \text { thick }} \equiv \Phi_{\mathrm{i} / \mathrm{T}}=1.482 \Phi_{1}$, vortices occupy all the pinning sites and interstitial positions inside each thick rhombus, one vortex per each thick rhombus. The parameters are $f_{p} / f_{0}=2.0, r_{p}=0.1 \lambda$.

ber of thin rhombuses. However, some of the thin rhombuses are separated from other thin rhombuses by thick ones (call them single thin rhombuses), but some of them have common sides with each other (double thin rhombuses). Therefore, the number of vacancies (i.e., unoccupied pins) is then the number of single thin rhombuses plus one half of the number of "double" thin rhombuses,

$$
N_{p}^{\mathrm{un}}\left(\Phi_{\mathrm{v} / \mathrm{t}}\right)=N_{\mathrm{rh}}^{\mathrm{s}}+\frac{1}{2} N_{\mathrm{rh}}^{\mathrm{d}}
$$

where $N_{p}^{\text {un }}$ is the number of unoccupied pinning sites at $\Phi=\Phi_{\mathrm{v} / \mathrm{t}}, N_{\mathrm{rh}}^{\mathrm{s}}$ and $N_{\mathrm{rh}}^{\mathrm{d}}$ are the numbers of single and double thin rhombuses, correspondingly.

For higher vortex densities (e.g., for $\Phi=$ $\left.\Phi_{\text {interstitial } / \text { thick }} \equiv \Phi_{\mathrm{i} / \mathrm{T}}=1.482 \Phi_{1}\right)$, we can expect the appearance of a feature (maximum) of the function $J_{c}(\Phi)$ related to the entry of a single interstitial vortex into each thick rhombus. The position of this maximum is determined by the number of vortices at $\Phi=\Phi_{1}$, which is $N_{v}(\Phi)=N_{p}$, plus the number of thick rhombuses, $N_{\mathrm{rh}}^{\text {thick }}$. Since the ratio of the number of thick to that of thin rhombuses is the golden mean, $\tau=(1+\sqrt{5}) / 2$ (in an infinite lattice; in a finite lattice, it is determined by a ratio of two successive Fibonacci numbers), then $N_{\mathrm{rh}}^{\text {thick }}=N_{\mathrm{rh}} / \tau$, where $N_{\mathrm{rh}}$ is the total number of rhombuses. Here we used: $1 / \tau=\tau-1$.

In Fig. 9a, the function $J_{c}(\Phi)$ is plotted for an array of pinning sites in the form of a part of the Penrose lattice, shown in Figs. 9c,d,e, which consists of 20 thick $\left(N_{\mathrm{rh}}^{\text {thick }}=\right.$
20) and 15 thin $\left(N_{\mathrm{rh}}^{\text {thin }}=15\right)$ rhombuses and contains 46 nodes (pinning sites). The nodes are connected by lines in order to show the rhombuses.

The distribution of vortices for $\Phi=\Phi_{1}$ is shown in Fig. 9c. The number of vortices $N_{v}$ coincides with the number of pinning sites $N_{p}$, and almost all the vortices are pinned. Note that since we use a square simulation cell, some of the vortices are always outside the "Penrose sample". These vortices mimic the externally applied magnetic field and determine the average vortex density in the entire simulation cell. Because of these additional vortices, the value of the function $J_{c}(\Phi)$ effectively reduces approximately by a "filling" factor $\eta$ which is

$$
\eta=\frac{A_{P}}{A} \approx 0.575
$$

Here $A_{P}$ and $A$ are the areas of the Penrose lattice (i.e., the area of all the rhombuses) and of the simulation region.

The value of the function $J_{c}(\Phi)$ in the maximum $\Phi=\Phi_{1}$ (Fig. 9a) is $J_{c} \approx 0.55$, i.e. corresponds to almost perfect matching (two pinning sites occurred to be unoccupied in the distribution shown in Fig. 9c) taking into account Eq. (16).

Let us now more carefully analyze the calculations of $J_{c}(\Phi)$ for the Penrose lattice, presented in Fig. 9. In Fig. $9 \mathrm{~b}$, the distribution of vortices is shown for $\Phi=$ $\Phi_{\mathrm{v} / \mathrm{t}}$. Vortices occupy all the pinning sites except those situated in one of the two vertices, connected by the short diagonal, of each thin rhombus. Thus, each single and each pair of double thin rhombuses contain one vacancy (unoccupied pinning site) at the matching field $\Phi_{\mathrm{v} / \mathrm{t}}$. The corresponding maximum is indicated by the arrow (b) in Fig. 9a.

The location of vortices for $\Phi=\Phi_{1}$ (the maximum (c) in Fig. 9a) is shown in Fig. 9c; here the number of vortices $N_{v}$ coincides with the number of pinning sites $N_{p}$, and almost all the vortices are pinned.

The distribution of vortices for $\Phi=\Phi_{\mathrm{i} / \mathrm{T}}$ (Fig. 9d) is also in agreement with our expectation: vortices occupy all the pinning sites (there is only a single "defect" in the distribution shown in Fig. 9d: one vortex left the pinning site and became interstitial) plus interstitial positions inside each thick rhombus, i.e., one vortex per each thick rhombus. However, the corresponding feature of the function $J_{c}(\Phi)$ (arrow (d) in Fig. 9a) is less pronounced than the two above maxima at $\Phi=\Phi_{1}$ and at $\Phi=\Phi_{\mathrm{v} / \mathrm{t}}$.

In addition, there is a weak feature of the function $J_{c}(\Phi)$ at $\Phi \approx \Phi_{\mathrm{v} / \mathrm{t}} / 2$, which more clearly manifests itself for larger Penrose-lattice pinning arrays (see Fig. 10a).

Therefore, the calculated distributions of the vortices pinned on the Penrose-lattice pinning site array and the resulting function $J_{c}(\Phi)$ have revealed the QP features which are in agreement with our expectations. The specific structure of the function $J_{c}(\Phi)$ is consistent with two previous derivations both based on continuously deforming a QP lattice into a Penrose one (Sections V and 
FIG. 10: (This figure is available in "png" format: "Penrose Fig 10.png"; color) The critical depinning current $J_{c}$ as a function of the applied magnetic flux, $\Phi \sim N_{v}$, for an array of pinning sites placed in the nodes of a five-fold Penrose lattice (for $N_{p}=301$ ) (a). The distributions of vortices (shown by green solid circles) pinned on the Penrose-lattice pinning site array (shown by red open circles), for specific values of the applied magnetic flux which correspond to two matching fields: (b) $\Phi=\Phi_{\mathrm{v} / \mathrm{t}}$, vortices occupy all the pinning sites except one in each thin rhombus; (c) $\Phi=\Phi_{1}$, all the vortices are pinned. The parameters are the same as in Fig. 9.

\section{$\mathrm{VI})$.}

In Fig. 10a, the function $J_{c}\left(N_{v}\right)$ is shown for a larger Penrose-lattice array of pinning sites, $N_{p}=301$. The above QP features in $J_{c}\left(N_{v}\right)$ are much more pronounced in this case then for smaller arrays because of a considerable reduction of the "noise" related with an entry of each single vortex in the system.

In particular, the main maximum of the function $J_{c}\left(N_{v}\right)$, which corresponds to the matching condition $\Phi=\Phi_{1}\left(N_{v}=301\right)$, transforms into a rather sharp peak with the magnitude $\eta$. Also, a local maximum of $J_{c}(\Phi)$ at $\Phi \approx \Phi_{\mathrm{v} / \mathrm{t}} / 2$, is more pronounced for $N_{p}=301$, as mentioned above.

Finally, Fig. 11 demonstrates the function $J_{c}(\Phi)$, calculated for different samples with $N_{p}=46,141$, and 301 (Fig. 11a), and also for different criteria of $J_{c}$ : for the "static" and dynamical criteria (Fig. 11b). In the dynamical simulations of $J_{c}$ using a threshold criterion, i.e., $J_{c}$ is obtained as the minimum current $J \propto f_{\mathrm{i}}^{d}$ which depins the vortices. In the Appendix A, we show onset of vortex motion when the applied current $J$ exceeds the critical currnet: $J>J_{c}$. The results obtained using these two criteria are essentially equal, and throughout this work we use the "static" criterion defined above.

\section{ANALYTICAL APPROACH}

The following analysis could provide a better understanding of the above structure of $J_{c}(\Phi)$ for the Penrose pinning lattice. Let us compare the elastic $E_{\mathrm{el}}$ and pinning $E_{\text {pin }}$ energies of the vortex lattice at $H_{1}$ and at (the lower field) $H_{\mathrm{v} / \mathrm{t}}$, corresponding to the two maxima of $J_{c}$ (e.g., Figs. 9a, 10a, 11). Vortices can be pinned if the gain $E_{\text {pin }}=U_{\text {pin }} \beta n_{\text {pin }}$ of the pinning energy is larger than the increase of the elastic energy [31, 32, 33] related to local compressions:

$$
E_{\mathrm{el}}=C_{11} \frac{\left(a_{\mathrm{eq}}-b\right)^{2}}{a_{\mathrm{eq}}} .
$$

The shear elastic energy $\left(\propto C_{66}\right)$ provides the same qualitative result. Here, $U_{\text {pin }} \sim f_{p} r_{p}, n_{\text {pin }}$ is the density of pinning centers,

$$
\beta\left(H \leq H_{1}\right)=H / H_{1}=B /\left(\Phi_{0} n_{\text {pin }}\right),
$$
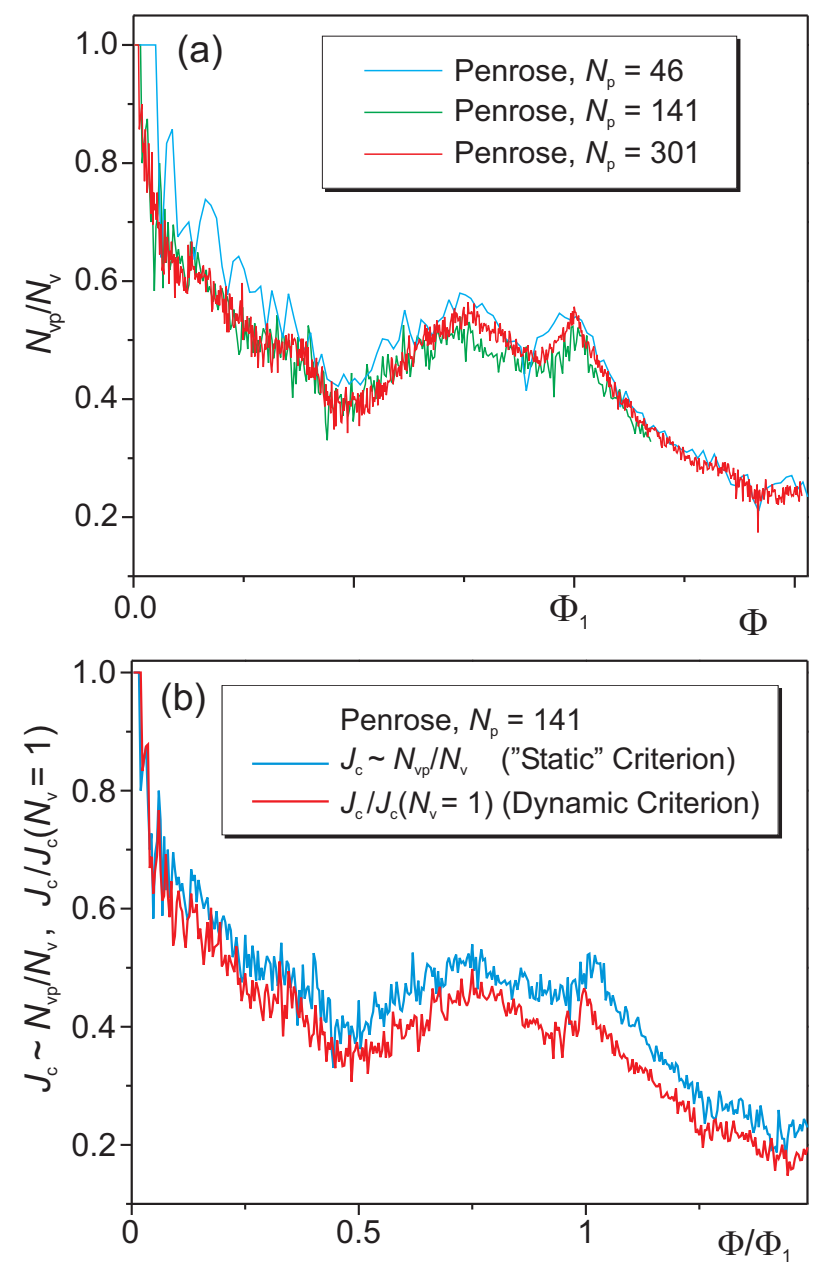

FIG. 11: (Color) (a) The critical depinning current $J_{c}$ as a function of the applied magnetic flux, $\Phi \sim N_{v}^{(p)} / N_{v}$, shown for three different Penrose-lattice pinning site arrays: $N_{p}=$ 46 (shown by blue solid line), $N_{p}=141$ (shown by green solid line), $N_{p}=301$ (shown by red solid line). The revealed features are hallmarks of a Penrose-lattice pinning site array. (b) Comparison of $J_{c}$ as a function of the applied magnetic flux, calculated using the "static" and dynamical criteria. The latter means calculating $J_{c}$ using a threshold criterion, i.e., $J_{c}$ is obtained as the minimum current $J \propto f_{\mathrm{i}}^{d}$ which depins the vortices. The results obtained using these two criteria are essentially equal.

and $\beta\left(H>H_{1}\right)=1$ is the fraction of occupied pinning sites $\left(\beta=1\right.$ for $H=H_{1}$, and $\beta=0.757$ for $\left.H=H_{\mathrm{v} / \mathrm{t}}\right)$, $a_{\mathrm{eq}}=\left(2 / \sqrt{3} \beta n_{\text {pin }}\right)^{1 / 2}$ is the equilibrium distance between vortices in the triangular lattice, $b$ is the minimum distance between vortices in the distorted pinned vortex lattice $\left(b=a / \tau\right.$ for $H=H_{1}$ and $b=a$ for $\left.H=H_{\mathrm{v} / \mathrm{t}}\right)$, and

$$
C_{11}=\frac{B^{2}}{4 \pi\left(1+\lambda^{2} k^{2}\right)}
$$

is the compressibility modulus for short-range deformations 31] with characteristic spatial scale $k \approx\left(n_{\text {pin }}\right)^{1 / 2}$. 
The dimensionless difference of the pinning and elastic energies is

$$
E_{\text {pin }}-E_{\mathrm{el}}=\frac{\beta f_{\text {diff }} n_{\text {pin }} \Phi_{0}^{2}}{4 \pi \lambda^{2}}
$$

where

$$
f_{\text {diff }}=\frac{4 \pi \lambda^{2} U_{\text {pin }}}{\Phi_{0}^{2}}-\beta\left[1-b\left(\frac{\beta \sqrt{3} n_{\text {pin }}}{2}\right)^{1 / 2}\right]^{2} .
$$

The function $f_{\text {diff }} 0$ is shown schematically in the inset to Fig. 12. Near matching fields, $J_{c}$ has a peak when $f_{\text {diff }}>0$ (and no peak when $f_{\text {diff }}<0$ ). Since only two matching fields provide $f_{\text {diff }}>0$, then our analysis explains the two-peak structure observed in $J_{c}$ shown in Figs. 9a, 10a, 11. For instance, for the main matching fields Eq. (20) gives: $f_{\text {diff }}\left(\Phi_{\mathrm{v} / \mathrm{t}}\right) \approx 0.0056, f_{\text {diff }}\left(\Phi_{1}\right) \approx$ 0.0058 , and $f_{\text {diff }}\left(\Phi_{\mathrm{i} / \mathrm{T}}\right) \approx-0.09$. Note that for weaker pinning, the two-peak structure gradually turns into one very broad peak, and eventually zero peaks for weak enough pinning (see Fig. 12). The $J_{c}$ peaks corresponding to higher matching fields are strongly suppressed because of the fast increase $\left(\propto B^{2}\right)$ of the compressibility modulus $C_{11}$ and, thus, the elastic energy with respect to the pinning energy; the latter cannot exceed the maximum value $U_{\text {pin }} n_{\text {pin }}$. The subharmonic peaks of $J_{c}$, which could occur for lower fields $H<H_{\mathrm{v} / \mathrm{t}}$, are also suppressed due to the increase of $C_{11}$ associated with the growing spatial scales $1 / k$ of the deformations.

\section{THE CRITICAL CURRENT $J_{c}(\Phi)$ IN A RANDOMLY DISTORTED TRIANGULAR LATTICE}

Above we have studied the function $J_{c}(\Phi)$ for periodic, QP 2D arrays of pinning sites and analyzed the transition from the periodic triangular lattice to the QP Penrose lattice (see Fig. 7). One of the issues, which is related to this analysis and can be useful for practical applications, is the increase of the critical current (shown, e.g., in Fig. 7f) in the regions corresponding to minima of $J_{c}(\Phi)$ for periodic (triangular) pinning arrays. The situation shown in Fig. $7 \mathrm{f}$ seems to be the optimal from the point of view of a homogeneous increase of $J_{c}(\Phi)$ without degradation of the main peak at $\Phi=\Phi_{1}$. Recall that it corresponds to a slightly "quasiperiodically distorted" triangular lattice (see Fig. 7b). The pinning sites of the triangular lattice are shifted from their "correct" positions but not randomly: their positions are determined by vortex-vortex interaction, which tries to restore the triangular lattice, and by the memory about previous configurations including the initial one, i.e., the Penrose lattice. Analyzing the lattice presented in Fig. 7b we can deduce that it keeps some short-range order (i.e., distorted triangular cells similar in shape and size to those in the triangular lattice) but does not have long-range order of the triangular lattice. As a result, the main peak

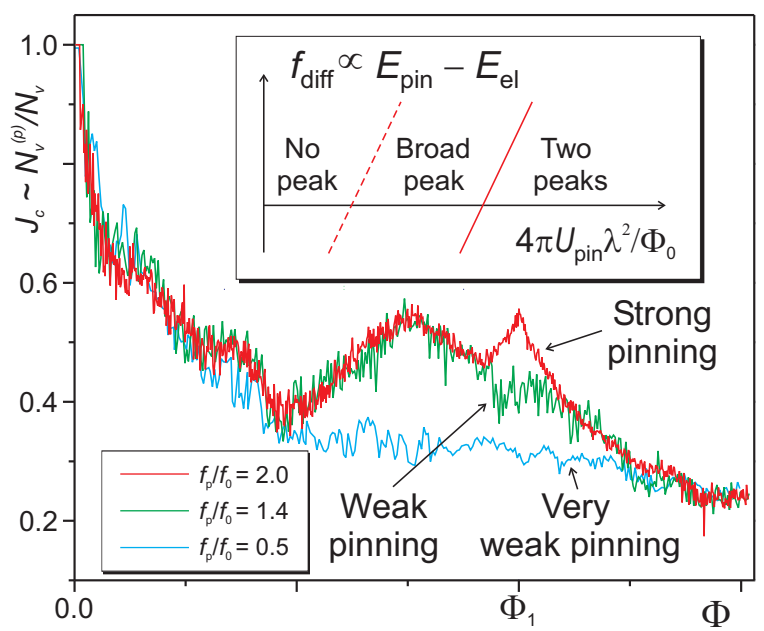

FIG. 12: (Color) The critical current $J_{c}(\Phi)$ for Penroselattice arrays for different pinning strength $f_{p} / f_{0}=2.0$ (red solid line), $f_{p} / f_{0}=1.4$ (green solid line), $f_{p} / f_{0}=0.5$ (blue solid line). The peak at $\Phi_{1}$ is suppressed for weaker pinning $\left(f_{p} / f_{0}=1.4\right)$. Eventually, all the main peaks disappear for sufficiently weak pins $\left(f_{p} / f_{0}=0.5\right)$. The inset shows the dimensionless difference, $f_{\text {diff }}$, of the pinning and the elastic energies versus the pinning-to-interaction energy ratio, for the broad $J_{c}$ peak at $\Phi_{\mathrm{v} / \mathrm{t}}$ (red dashed line) and for $\Phi_{1}$ (red solid line). Only $f_{\text {diff }}>0$ gives stable peaks in $J_{c}$.

remains, since it is related to the short-order matching effects (i.e., over the distances of the order inter-site spacings $a$ ). The sharp decrease around the maximum and appearance of the deep valleys is explained by the absence (due to long-range order, i.e., over distances longer than $a$ ) of any matching effects for the flux densities close to $\Phi=\Phi_{1}$ (there is no matching for $\Phi=\Phi_{1} / 2$ or $\Phi=1.5 \Phi_{1}$ and $\Phi=2 \Phi_{1}$ for the triangular lattice). When the long-range order is destroyed, as shown in Fig. 7b, matching effects other than $\Phi=\Phi_{1}$ become allowed.

It is appropriate to mention here that the QP Penrose lattice possesses a short-range order but does not have a long-range translational order. In such a way, the "quasiperiodically distorted" triangular lattice (Fig. 7b) or the QP Penrose lattice itself are good candidates for the optimal enhancement of the critical current in the regions where the function $J_{c}(\Phi)$ have minima for a periodic lattice. (It should be noted here that the curves shown, e.g., in Fig. 7h, for the triangular and the Penrose lattices are calculated for the same cell, although the effective area of the Penrose lattice is smaller than that of the triangular lattice with the same number of pinning sites. This discrepancy is taken into account by the "filling factor" introduced by Eq. (16). It should be also recalled, when comparing the function $J_{c}(\Phi)$ for the case of the triangular and the Penrose lattices, that the main maximum of the curve for the Penrose lattice (see Fig. 11) is the second sharp peak at $\Phi=\Phi_{1}$.)

In this respect, it is interesting to compare the above 
FIG. 13: (This figure is available in "png" format: "Penrose Fig 13.png"; color) Left column: Randomly distorted triangular lattices for $r_{r a n}^{\max }=0.2(a / 2)(\mathrm{a}), 0.3(a / 2)(\mathrm{b}), 0.4(a / 2)(\mathrm{c})$, and for $a / 2(\mathrm{~d})$. For comparison, the triangular lattice is also shown. Right column: The functions $J_{c}(\Phi)$ corresponding to the distributions of pinning sites shown in (a) to (d), are presented, respectively, in (e) to (h). At low levels of noise (e), (f), the valleys (minima) start to fill due to disappearance of the long-range order, similarly to the case of the Penrose lattice, although accompanied with a weaker enhancement of $J_{c}$ than for the case of the "quasiperiodic distortion" (Fig. 7f). For higher levels of noise, the main peak degrades without any essential enhancement of $J_{c}$ in the neighborhood (g), (h).

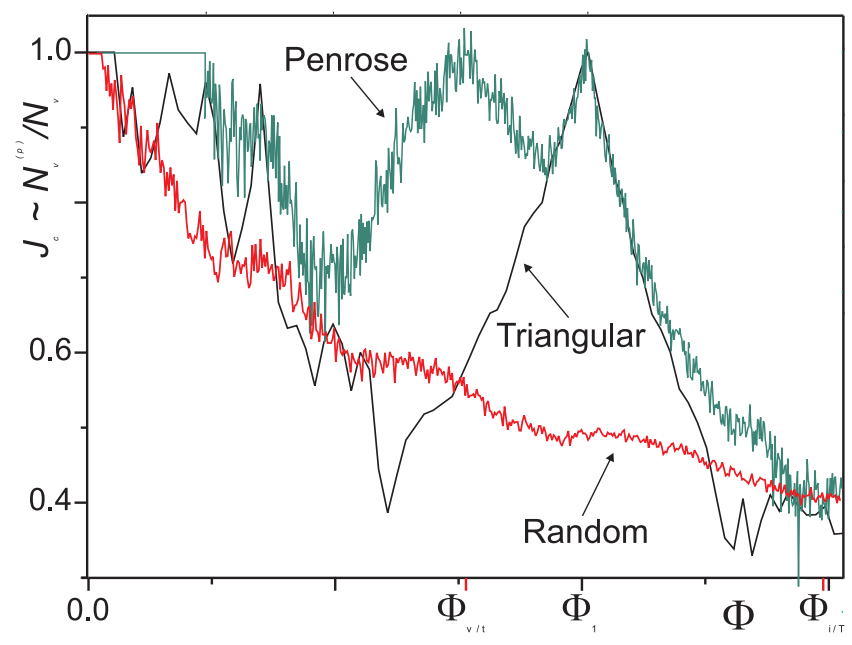

FIG. 14: (Color) The critical current $J_{c}(\Phi)$ for a 301-sites Penrose-lattice (dark green solid line), (recalculated for flux only on the Penrose area, $A_{P}$ ), triangular (black solid line) and random (red solid line) pinning arrays. The Penrose lattice provides a remarkable enhancement of $J_{c}(\Phi)$ over a very wide range of values of $\Phi$ because it contains many periods in it.

results for the "quasiperiodic distortion" of the triangular lattice with its random distortion. For this purpose, we introduce a random angle $\alpha_{\text {ran }}: 0<\alpha_{\text {ran }}<2 \pi$, and a random radius of the displacement $d_{\text {ran }}: 0<r_{\text {ran }}<r_{\text {ran }}^{\max }$, where $r_{\text {ran }}^{\max }$ is the maximal displacement radius, which is a measure of noise measured in units of $a / 2$, where $a$ is the (triangular) lattice constant.

In Fig. 13, randomly distorted triangular lattices are shown for $r_{\text {ran }}^{\max }=0.2(a / 2)$ (Fig. 13a), 0.3(a/2) (Fig. 13b), $0.4(a / 2)$ (Fig. 13c), and for $a / 2$ (Fig. 13d). For comparison, the triangular lattice is also shown in Figs. 14a to d. The corresponding functions $J_{c}(\Phi)$ are presented, respectively, in Figs. 13e to h. At low levels of noise (e.g., Fig. 13e, f) the valleys (minima) start to fill due to the disappearance of the long-range order, similarly to the case of the Penrose lattice, although accompanied with a weaker enhancement of $J_{c}$ than for the case of the "quasiperiodic distortion" (Fig. 7f). For higher levels of noise, the main peak degrades without any essential enhancement of $J_{c}$ in the neighborhood (Figs. 13g, h).

For comparison, we also show in Fig. 14 the $J_{c}(\Phi)$ for a Penrose-lattice (calculated for the sample with $N_{p}=301$, only for the area of the Penrose lattice $A_{P}$, see Eq. (16)). Notice that the QP lattice leads to a very broad and potentially useful enhancement of the critical current $J_{c}(\Phi)$, even compared to the triangular or random APS. The remarkably broad maximum in $J_{c}(\Phi)$ is due to the fact that the Penrose lattice has many (infinite, in the thermodynamic limit) periodicities built in it 11]. In principle, each one of these periods provides a peak in $J_{c}(\Phi)$. In practice, like in quasicrystalline difraction patterns, only few peaks sre strong. This is also consistent with our study. Furthermore, the pinning parameters can be adjusted by using as pinning centers either antidots (microholes of different radii "drilled" in the film [1, 6], or blind antidots [7] of different depths and radii. Thus, our results could be observed experimentally.

\section{CONCLUSIONS}

The critical depinning current $J_{c}$, as a function of the applied magnetic flux $\Phi$, has been studied in QP pinning arrays, from one-dimensional chains to two-dimensional arrays of pinning sites set in the nodes of quasiperiodic lattices including a 2D-quasiperiodic triangular lattice and a five-fold Penrose lattice.

In a $1 \mathrm{D}$ quasiperiodic chain of pinning sites, positions of the peaks of the function $J_{c}(\Phi)$ are governed by "harmonics" of long and short periods of the quasiperiodic chain. Independently of the length of the chain, the peaks form a set of quasiperiodic sequence including a Fibonacci sequence as a basic subset. Analyzing the evolution of the peaks, when a continuous transition is performed from a periodic to a quasiperiodic lattice of the pinning sites, we found that the peaks related to the Fibonacci sequence are most pronounced when the ratio of lengths of the long and the short periods is the golden mean. A comparison of the sets of peaks for different chains shows that the functions $J_{c}(\Phi)$ for the $1 \mathrm{D}$ quasiperiodic chain is self-similar. In the $k$-space, the self-similarity effect is displayed in the Fourier-transform of the distribution function of the system of vortices pinned on a 1D quasiperiodic array of pinning centers. The evolution of quasiperiodic peaks when gradually changing the "quasiperiodicity" parameter $\gamma$ (i.e., ratio of the lengths of short to long elements of a quasiperiodic chain) has revealed a continuous transition from a periodic chain - through the set of quasiperiodic states - to another periodic chain with a longer period. This phenomena has been studied both in real space and in reciprocal $k$-space.

In $2 \mathrm{D}$ quasiperiodic pinning arrays (e.g., Penrose lattice), the pinning of vortices is related to matching 
conditions between a triangular vortex lattice and the quasiperiodic lattice of the pinning centers. Although more complicated than in $1 \mathrm{D}$ pinning chains, the specific behavior of $J_{c}(\Phi)$ is determined by the presence of two different kinds of elements - thick and thin rhombuses forming the quasiperiodic lattice. Based on these considerations, the positions of the main maxima of $J_{c}(\Phi)$ for Penrose lattice are predicted.

In particular, for the first matching field each pinning site is occupied by a vortex. The corresponding maximum of the function $J_{c}(\Phi)$ is broad since it involves at least three kinds of local matching effects of the flux lattice, with the rhombus side and with short diagonals of thick and thin rhombuses.

Another Penrose-lattice matching field is related with local matching effects which involve the intervortex distance of the vortex flux lattice, the rhombus side and the short diagonal of thick rhombus. For this field, all the pinning sites are occupied, which are situated in the vertices of thick rhombuses and only three out of four in the vertices of thin rhombuses. The number of unoccupied pinning sites is governed by the number of thin rhombuses. Some of the thin rhombuses are single (i.e., separated from other thin rhombuses by thick ones), while some of them are double (i.e., have common sides with each other). Therefore, the number of vacancies is the number of single thin rhombuses plus one half of the number of "double" thin rhombuses. One more important feature of the function $J_{c}(\Phi)$ occurs for higher vortex densities, when a single interstitial vortex enters each thick rhombus.

Numerical simulations performed for various sample sizes have revealed a good agreement with our predictions.

The revealed features can be more or less pronounced depending on specific relations between the vortex-vortex interaction constant and the strength of the pinning sites, as well as on the distance between pinning sites and their radius. While the vortex-vortex interaction constant is a material parameter, all others can be adjustable parameters in experiments with artificially created quasiperiodic pinning arrays. This can be reached by using, for instance, antidots (i.e., microholes "drilled" in a superconductor film) or blind antidots of different depths and radii as pinning centers. Our calculations provide the necessary relations between these parameters for possible experimental realizations.

A continuous deformation of the Penrose lattice to a periodic triangular lattice (i) shows that the above revealed features are hallmarks of quasiperiodic pinning arrays; (ii) provides us with a tool for the controlled change of the magnitude, sharpness and the position of the peaks of $J_{c}(\Phi)$ that is important for possible applications. In particular, our analysis shows that the quasiperiodic lattice provides an unusually broad critical current $J_{c}(\Phi)$, that could be useful for practical applications demanding high $J_{c}$ 's over a wide range of fields.

\section{ACKNOWLEDGMENT}

This work was supported in part by the National Security Agency (NSA) and Advanced Research and Development Activity (ARDA) under Air Force Office of Scientific Research (AFOSR) contract number F4962002-1-0334; and also supported by the US National Science Foundation grant No. EIA-0130383, and RIKEN's President's funds.

\section{APPENDIX A: Onset of vortex motion for currents higher then the critical current: $J>J_{c}$}

Here we present vortex flow patterns for currents exceeding the critical value, $J>J_{c}$. In Fig. 15 , the vortex flow patterns are shown calculated for a Penrose sample with $N_{p}=141$ (pinning sites shown by red circles). The ground-state vortex configuration is shown in Fig. 15a (vortices shown by green dots) for $\Phi \approx \Phi_{1}$ and when no driving force is applied. This vortex configuration is similar to those shown in Figs. 9c and 10c, when the number of vortices (within the sample area) is equal to the number of pinning sites, and all the vortices are pinned. When an increasing driving force $f_{d} \sim J$ is applied, the vortices do not move until $f_{d}$ reaches some threshold value, when vortices depin. The current which corresponds to the driving force depinning the vortices, is then defined as the critical current $J_{c}$ (dynamical criterion). A comparison of $J_{c}$ 's calculated using this criterion and the static criterion, is shown above in Fig. 11b. When unpinned, the vortices move along complicated trajectories, or "channels" created by pinning arrays and interacting with other vortices. Figs. 15b to $15 \mathrm{~d}$ show the onset of the flux motion for $J>J_{c}$, following the traces of moving vortices over distances about $0.5 \lambda(\mathrm{b}), 1 \lambda(\mathrm{c})$, and $2 \lambda(\mathrm{d})$. On the subsequent consecutive snapshots, vortices are shown by a sequence of consecutive open blue circles (and by blue solid circles for the last snapshot on each panel). These show dynamical configurations of the vortex lattice in motion. Note the appearance of local "rivers" of vortices moving along the channels between neighboring pinning sites. 
FIG. 15: (This figure is available in "png" format: "Penrose Fig 15.png"; color) Vortex flow patterns for $J>J_{c}$, calculated for a Penrose sample with $N_{p}=141$ (pinning sites shown by red circles). (a) Ground-state vortex configuration (vortices shown by green dots) when $\Phi \approx \Phi_{1}$, and no driving force is applied. (b-d) The onset of the flux motion for $J>J_{c}$, following the traces of moving vortices over distances about $0.5 \lambda(\mathrm{b}), 1 \lambda(\mathrm{c})$, and $2 \lambda(\mathrm{d})$. On the subsequent consecutive snapshots, vortex trajectories are shown by black dotted lines (the blue solid circles show the last snapshot on each panel).

[1] M. Baert, V.V. Metlushko, R. Jonckheere, V.V. Moshchalkov, and Y. Bruynseraede, Phys. Rev. Lett. 74, 3269 (1995).

[2] V.V. Moshchalkov, M. Baert, V.V. Metlushko, E. Rosseel, M.J. Van Bael, K. Temst, R. Jonckheere, and Y. Bruynseraede, Phys. Rev. B 54, 7385 (1996).

[3] J. Eisenmenger, P. Leiderer, M. Wallenhorst, and H. Dötsch, Phys. Rev. B 64, 104503 (2001).

[4] J. Eisenmenger, Z.-P. Li, W.A.A. Macedo, and I.K. Schuller, Phys. Rev. Lett. 94, 057203 (2005).

[5] J.E. Villegas, S. Savel'ev, F. Nori, E.M. Gonzalez, J.V. Anguita, R. García, and J.L. Vicent, Science 302, 1188 (2003); J.E. Villegas, E.M. Gonzalez, M.I. Montero, I.K. Schuller, J.L. Vicent, Phys. Rev. B 68, 224504 (2003); M.I. Montero, J.J. Akerman, A. Varilci, I.K. Schuller, Europhys. Lett. 63, 118 (2003).

[6] A.M. Castellanos, R. Wördenweber, G. Ockenfuss, A.v.d. Hart, and K. Keck, Appl. Phys. Lett. 71, 962 (1997); R. Wördenweber, P. Dymashevski, and V.R. Misko, Phys. Rev. B 69, 184504 (2004).

[7] L. Van Look, B.Y. Zhu, R. Jonckheere, B.R. Zhao, Z.X. Zhao, and V.V. Moshchalkov, Phys. Rev. B 66, 214511 (2002).

[8] A.V. Silhanek, S. Raedts, M. Lange, and V.V. Moshchalkov, Phys. Rev. B 67, 064502 (2003).

[9] R. Penrose, Bull. Inst. Math. Appl. 10, 226 (1974); R. Penrose, Math. Intelligencer 2 (1), 32 (1979).

[10] N.G. de Bruijn, Koninklijke Ned. Akad. Weten. Proc., Ser. A 84, 39 (1981); 84, 53 (1981).

[11] Quasicrystals, Ed. J.-B. Suck, M. Schreiber, P. Häussler (Springer, Berlin, 2002).

[12] R.K.P. Zia and W.J. Dallas, J. Phys. A: Math. Gen. 18, L341 (1985).

[13] M. Kohmoto, B. Sutherland, and C. Tang, Phys. Rev. B 35, 1020 (1987).

[14] F. Nori and J.P. Rodriguez, Phys. Rev. B 34, 2207 (1986).

[15] Q. Niu and F. Nori, Phys. Rev. Lett. 57, 2057 (1986).

[16] A. Behrooz, M.J. Burns, H. Deckman, D. Levine, B. Whitehead, and P.M. Chaikin, Phys. Rev. Lett. 57, 368 (1986); Y.Y. Wang R. Steinmann, J. Chaussy, R. Rammal, and B. Pannetier, Jpn. J. Appl. Phys. 26,
1415 (1987); K.N. Springer and D.J. Van Harlingen, Phys. Rev. B 36, 7273 (1987).

[17] F. Nori, Q. Niu, E. Fradkin, and S.-J. Chang, Phys. Rev. B 36, 8338 (1987).

[18] F. Nori and Q. Niu, Phys. Rev. B 37, 2364 (1988).

[19] Q. Niu and F. Nori, Phys. Rev. B 39, 2134 (1989).

[20] Y.-L. Lin and F. Nori, Phys. Rev. B 65, 214504 (2002).

[21] S.-N. Zhu, Y.-Y. Zhu, and N.-B. Ming, Science 278, 843 (1997).

[22] F. Domínguez-Adame, A. Sánchez, and Y.S. Kivshar, Phys. Rev. E 52, R2183 (1995).

[23] M. Torres, J.P. Adrados, J.L. Aragon, P. Cobo, and S. Tehuacanero, Phys. Rev. Lett. 90, 114501 (2003).

[24] F. Nori, Science 278, 1373 (1996); C. Reichhardt, C.J. Olson, J. Groth, S. Field, and F. Nori, Phys. Rev. B 52, 10441 (1995); B 53, R8898 (1996); B 54, 16108 (1996); B 56, 14196 (1997);

[25] C. Reichhardt, C.J. Olson, and F. Nori, Phys. Rev. B 57, 7937 (1998).

[26] C. Reichhardt, C.J. Olson, and F. Nori, Phys. Rev. Lett. 78, 2648 (1997); B 58, 6534 (1998).

[27] B.Y. Zhu, F. Marchesoni, V.V. Moshchalkov, and F. Nori, Phys. Rev. B 68, 014514 (2003); Physica C 388389, 665 (2003); Physica C 404, 260 (2004); B.Y. Zhu, L. Van Look, F. Marchesoni, V.V. Moshchalkov, and F. Nori, Physica E 18, 322 (2003); B.Y. Zhu, F. Marchesoni, and F. Nori, Phys. Rev. Lett. 92, 180602 (2004); Physica E 18, 318 (2003); F. Marchesoni, B.Y. Zhu, and F. Nori, Physica A 325, 78 (2003).

[28] M. Kolár and F. Nori, Phys. Rev. B 42, 1062 (1990).

[29] K. Harada, O. Kamimura, H. Kasai, T. Matsuda, A. Tonomura, and V.V. Moshchalkov, Science 274, 1167 (1996).

[30] Y. Togawa et al. Phys. Rev. Lett. 95, in press (2005).

[31] E.-H. Brandt J. Low Temp. Phys. 26, 709, 735 (1977); Phys. Rev. B 34, 6514 (1986); Rep. Prog. Phys. 58, 1465 (1995).

[32] G. Blatter, M.V. Feigel'man, V.B. Geshkenbein, A.I. Larkin, and V.M. Vinokur, Rev. Mod. Phys. 66, 1125 (1994).

[33] J.R. Clem, M.W. Coffey, Phys. Rev. B 46, 14622 (1992). 
This figure "Penrose_Fig_7.png" is available in "png" format from: http://arxiv.org/ps/cond-mat/0511098v1 
This figure "Penrose_Fig_9.png" is available in "png" format from: http://arxiv.org/ps/cond-mat/0511098v1 
This figure "Penrose_Fig_10.png" is available in "png" format from: http://arxiv.org/ps/cond-mat/0511098v1 
This figure "Penrose_Fig_13.png" is available in "png" format from: http://arxiv.org/ps/cond-mat/0511098v1 
This figure "Penrose_Fig_15.png" is available in "png" format from: http://arxiv.org/ps/cond-mat/0511098v1 